\title{
Amelioration of Leigh syndrome induced by mouse blastocyst complementation with a mutant human mitochondrial ATP synthase 6
}

Huijun Yuan ( $\sim$ HYuan@med.miami.edu )

University of Miami

Keith Webster

Baylor college of medicine

Tariq Bhatti

Mayo Clinic

William Hauswirth

HSC University of Florida

Alfred Lewin

University of Florida

John Guy

University of Miami

Article

Keywords: mito-targeted AAV, mutant m.8993T>G ATP6, Leigh Syndrome and Neuropathy, Ataxia and 49 Retinitis Pigmentosa (NARP)

Posted Date: October 21st, 2021

DOI: https://doi.org/10.21203/rs.3.rs-963913/v1

License: (c) (1) This work is licensed under a Creative Commons Attribution 4.0 International License.

Read Full License 
2 Amelioration of Leigh syndrome induced by mouse blastocyst complementation with a mutant 3 human mitochondrial ATP synthase 6

4

5 Huijun Yuan ${ }^{1,5^{*}}$, Keith A. Webster ${ }^{1,2}$, M. Tariq Bhatti ${ }^{3}$, William W. Hauswirth ${ }^{4}$, Alfred S. Lewin ${ }^{4}$, and John 6 Guy $^{1 *}$

7

8

10

11

12

13

14

16

17

18

19

20

21

22

23

24

25

26

27

28

29

30

1

2

${ }^{1}$ Bascom Palmer Eye Institute, University of Miami Miller School of Medicine, Miami, FL 33136, USA

${ }^{2}$ Cullen Eye Institute, Department of Ophthalmology, Baylor College of Medicine, Houston, TX 77030, USA.

Everglades Biopharma, LLC, Houston, TX 77030, USA

${ }^{3}$ Mayo Clinic, Rochester, MN 55902, USA

${ }^{4}$ University of Florida, College of Medicine, Gainesville, FL 32611, USA

${ }^{5}$ Division of Neonatology and Batchelor Children's Research Institute, Department of Pediatrics, University of Miami Miller School of Medicine, Miami, FL 33136, USA (Current)

*Senior author Dr. John Guy passed away as this manuscript was being revised. Correspondence and requests for materials should be addressed to H.J.Y (email: hyuan@med.miami.edu).

Abbreviations:

MRI: magnetic resonance imaging; PERG: pattern electroretinogram; FERG: flash electroretinogram; TEM: Transmission electron microscopy; OXPHOS: oxidative phosphorylation; RGC: retinal ganglion cell; INL: inner nuclear layer; ONL: outer nuclear layer. 
32 Mutations in mitochondrial genes are primary causes of multiple inherited disorders, especially affecting 33 tissues with high aerobic energy demands such as the eye, brain, heart, and skeletal muscles. Such 34 mitochondrial diseases are untreatable, have poor prognoses, and relevant animal models are few. Here we 35 microinjected into the mouse blastocyst an adeno-associated virus (AAV) that we redirected to 36 mitochondria to deliver the mutant human adenosine triphosphate (ATP) synthase subunit 6 gene 37 (m.8993T $>\mathrm{G})$ responsible for Maternally Inherited Leigh Syndrome, a neurologic disease renowned for causing rapidly fatal encephalomyelopathy in childhood or Neuropathy, Ataxia and Retinitis Pigmentosa in adults. Mice bred over six generations exhibited hallmarks of the human disease with one or multiple systemic symptoms including early death, paralysis, hunching, vision loss and seizures. Necropsy revealed

41 spongiform encephalopathy, retinal degeneration, and cardiomegaly. Intravenous injection of a 42 mitochondrially directed AAV serotype 9 vector containing wild-type ATP6 after disease onset, conferred 43 prolonged survival and reduced paralysis. When administered prior to disease onset, all treated mice 44 survived, and visual and motor function improved. These results support further development of gene 45 therapy strategies involving intravenous delivery of mito-targeted AAV9. 
54 Leigh syndrome (LS) is a progressive neurodegenerative disease that affects as many as 1 in 40,000 live

55 births $^{1,2}$. It is caused by mutations in nuclear or mitochondrial genes that encode structural components or 56 assembly factors of the electron transport chain and ATP synthase complexes I-V that are responsible for 57 eukaryotic energy production by oxidative phosphorylation (OXPHOS). Over 75 loci on more than 60 58 genes affecting complexes I-V have been linked to LS with about $25 \%$ of cases caused by mutations of 59 mitochondrial DNA (mtDNA) $)^{3}$ LS-associated mtDNA mutations include 6 genes in complex I, COX-III 60 in Complex IV, and the ATP6 subunit of Complex $\mathrm{V}^{1,3}$. Because all mammalian cells contain numerous 61 mitochondria, and correspondingly numerous mt-genomes, mutated and wild type mtDNA can coexist in 62 conditions known as heteroplasmy. In general, symptoms of disease only manifest when the ratios of mutated to wild type mtDNA are increased to levels that impair OXPHOS sufficiently to restrict physiological functions.

The typical onset of LS is 3-12 months with $83 \%$ of cases presenting by age $2 \mathrm{yr}$ and evidence of congenital abnormalities in $22 \%$ of cases $^{1,3}$. Although there is more variability in adult-onset LS, the core features are

67 similar. LS subjects typically present with closely similar brain MRI scans that identify symmetrical 68 necrotizing lesions of the basal ganglia, thalamus, brainstem and cerebellum ${ }^{1,3}$. Symptoms vary and can 69 include psychomotor arrest or decline, hypotonia, spasticity, ataxia, dystonia, lethargy, seizures, infantile 70 spasms, movement disorders, dysphagia, abnormal ocular movements, hearing loss and respiratory failure $71{ }^{1,4,5}$. Mouse models with mutations or deletions of OXPHOS-related genes that mimic the human LS 72 condition have been described for both nuclear and mitochondrial origin genes ${ }^{1,6-11}$. These mouse models 73 have brain MRI profiles that closely mimic human LS, and symptoms that include progressive 74 encephalopathy, growth retardation, hypothermia, ataxia, hypotonia, lethargy, failure to thrive, breathing 75 irregularities and early death.

76 There is no cure for LS or indeed any of the up to 300 other mitochondrial diseases that have been

77 described ${ }^{1,12}$ although there is evidence for successful palliative pharmacological treatments, and molecular 78 genetic therapies to correct or mitigate the effects of the mutations are in the pipeline $e^{1,4,8,12}$. Mouse models 
have been developed to mimic LS associated with nuclear gene mutations in complexes I, II, and IV, the

80

81

82 most common being the complex I NDUFS4 knockout mouse that replicates multiple traits of human LS and has been instrumental in developing therapies ${ }^{4,8,13-16}$. Our group recently described a mouse model that mimics Leber hereditary optic neuropathy (LHON), a mitochondrial disease cause by mutations of the mitochondrial encoded NADH ubiquinone oxidoreductase subunit 4 (ND4) gene. Mouse germline delivery of a mutant human ND4 gene in an AAV vector targeted to the mitochondria produced mice with hallmark symptoms of LHON that were significantly reversed by subsequent intravitreal delivery of the wild type ND4 gene ${ }^{9}$.

Here we describe mouse germline delivery of a mutant ATP6 gene, responsible for the most common form of maternally inherited LS (MILS) and related Neuropathy, Ataxia and Retinitis Pigmentosa (NARP). Progeny of mice created by blastocyst transduction of a human mitochondrial-targeted mutant ATP6 AAV containing a $\mathrm{T}$ to $\mathrm{G}$ transition that causes substitution of arginine for leucine at amino acid 156 of the ATP6 gene, developed multiple system disorders including premature death, paralysis, vision loss, seizures, and cardiomegaly. To varying degrees, these hallmarks of human MILS were passed along the maternal lineage for six generations. Symptoms were partially mitigated by intravenous administration of a mito-targeted AAV9 containing the wild-type human ATP6 allele delivered either prior to or after disease onset.

\section{Results}

\section{Development of transgenic ATP6 (A6) mice by blastocyst injection of mitochondrially directed AAV2} carrying mutant human m.8993T $>$ G ATP6. The mitochondrial double membrane has proven to be a formidable barrier to manipulating the mitochondrial genome. Still, viruses ${ }^{17}$, linearized and doublestranded DNA appear to traverse them ${ }^{18}$ and express exogenous mitochondrial genes ${ }^{19}$. To generate a mutant A6 mouse, we created the mutant human m.8993T $>$ G ATP6 gene by site-directed mutagenesis of sc-HSP-ATP6 plasmid, which ATP6 gene was synthesized and fused a FLAG epitope followed by a stop codon (without a tRNA) ${ }^{20}$ and mCherry using the mitochondrial genetic code, into a self-complementary AAV serotype 2, (scAAV) plasmid (sc-HSP-mutATP6FLAG+mCherry) (Fig. 1a, described in methods). 
To direct the vector to mitochondria, we appended a cytochrome oxidase subunit 8 (COX8) pre-sequence into the $\mathrm{N}$ terminus of one of the three AAV capsid proteins (VP2) ${ }^{10}$. Three plasmids including modified pVP2cox8, sc-HSP-ATP6mutFLAG+mCherry, and pDG with mutations at positions 444, 500 and 730 (Y444F, Y500F, Y730F) to induce robust transduction ${ }^{21}$ were applied to produce the mitochondrial targeted scAAV2-mutATP6FLAG AAV2 viral vector. After packaging, mito-targeted scAAV2 aliquots were microinjected into mouse blastocysts and implanted into pseudo-pregnant females (Fig.1a). Of fifty-three founder mice generated by such blastocyst injections, six females with high ocular expression of mCherry, visualized by laser scanning ophthalmoscopy, were used to generate a total of 462 additional F1-F6 A6 mice. (Fig.1b, c \& Supplementary Table 1). The final A6 colony included 252 females, 203 males and 60 unsexed pups that died within 8 days of birth. The litter size mostly ranged from 8 to 12 pups. High early mortality rates were a major characteristic of all generations (Supplementary Table 2).

\section{Transgenic A6 mice develop human LS phenotypes including premature death, weight loss, paralysis,} hunching, seizures, off balance, tumors, and visual loss. Transgenic A6 mice presented a spectrum of human-like LS or NARP phenotypes that included premature death, weight loss, paralysis, hunching, seizures, motor disfunction, tumors, and ocular defects. From the entire colony of 515 transgenic mice, F0 through F6, such human-like LS/NARP features were recorded in $\sim 70 \%$ of animals (Supplementary Table 3). Of these, 167/515 (32\%) died before 15 months including 101/515 (20\%) that died before 6 months, revealing highly significant rates of premature death (Fig.1d, Supplementary Fig.1a \& Table 3). Six of 53 F0 mice died before 6 months of age, including four males that died before 3 months. F1 mice incurred the highest 6 -month mortality rate of $56 \%$, significantly greater than all other groups $(n=54 / 96)$, followed by an F2 mortality rate of 23\% that was also significantly greater than the F3-F5 groups. Of F0 to F6 mice that survived $>15$-months, at least $50 \%$ displayed one or more phenotypes of hunching, paralysis, motor disfunction (off-balance), visual loss or seizures (Supplementary Table 3). Hunching was observed in 11\% (38 of 350) A6 mice with a mean age 19.9 months (Supplementary Fig. 1). 3\% of all generation A6 mice developed severe hinder limb paralysis with a mean age 19.6 months (Fig. 1e, Supplementary Table $3 \&$ 
129 Video 1). For motor dysfunction, we focused on F0 and F1 generations (Supplementary Table 3). 130 Accelerating rotarod testing of ambulation and balance revealed latency-to-fall times for F0-F1 mice that 131 were significantly less than the corresponding times observed for similarly aged wild type mice (Fig. 1g,). 132 Of 68/101 (67\%) randomly selected F0 and F1 mice aged between 6-12 months, 59\% showed significant 133 motor disfunction (Supplementary Table 3). Seizures were observed in $0.8 \%$ of total F6 mice, with mean 134 age 22.5 months (Fig. 1f, Supplementary Table 3 \& Video 2). As noted in the Table 3 Legend, such seizure 135 estimates are likely low because mice were not under constant surveillance. Reduced weight gain was 136 another significant clinical feature (Supplementary Table 3). A6 mice that succumbed to premature death 137 at age $<6$ months had average body weights of $17 \mathrm{~g}$, significantly less than the corresponding mean weights 138 of age-matched wild types (25g), (Fig.1h). Combined assessment of ocular defects by pattern 139 electroretinogram (PERG), flash electroretinogram (ERG) and histology (H\&E) revealed significantly 140 defective ocular functions in 35 of $43(80 \%)$ A6 mice selected from F0-F2 generations (Supplementary 141 Table 3), included declines in 10 of 11 by PERG, (Fig. 1i, relative to wild types), and 5 of 11 founders by 142 FERG, (Fig. 1j, compared with wild types). Together the results support the presence of pronounced but 143 variable symptoms of A6 mice that mimic those reported for human MILS and NARP. In addition, at least $1447 \%$ of A6 mice developed tumors with a mean age 20.1 months (Supplementary Table 3 \& Fig. 6). 
a

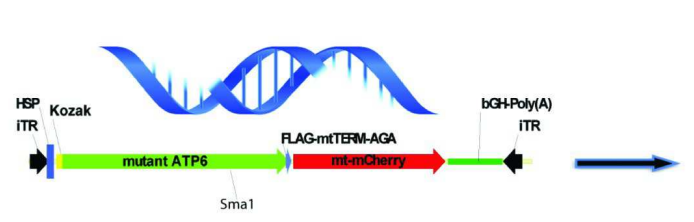

MTS-AAVs
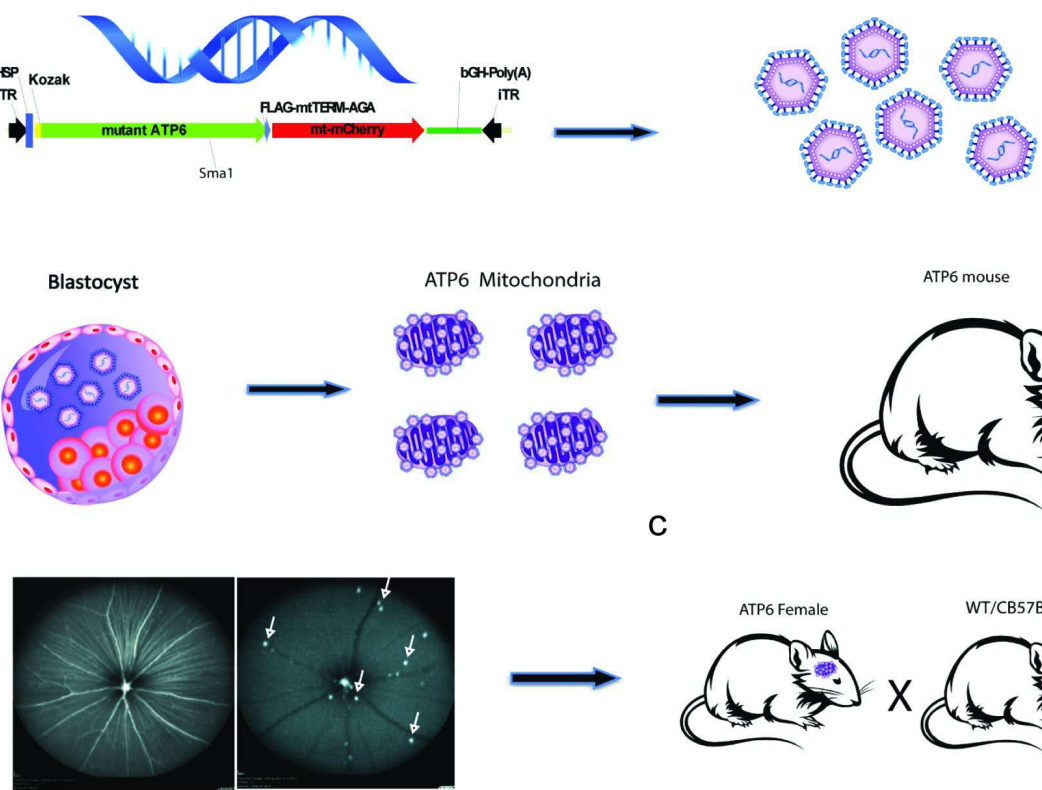

ATP6 Mitochondria

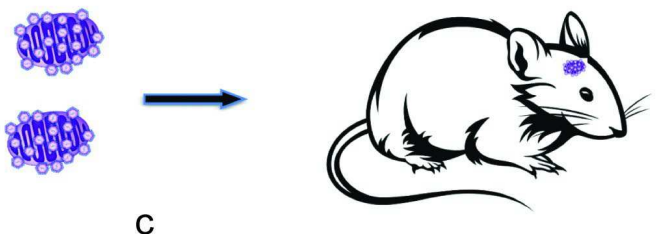

C

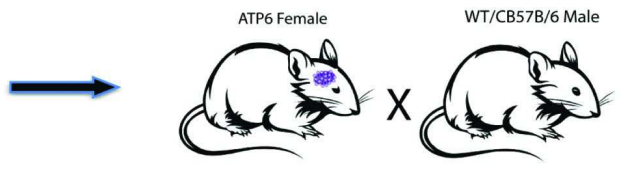

e
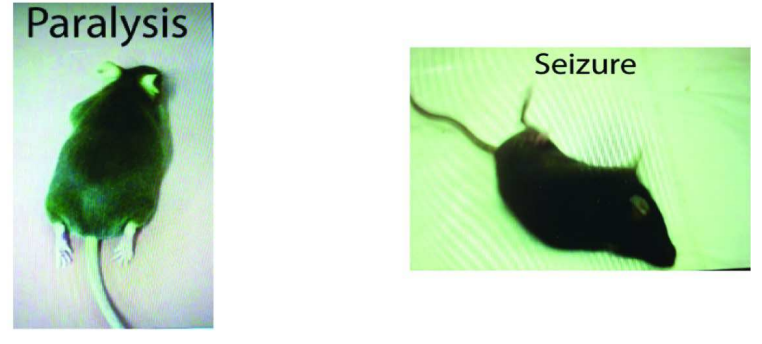

g

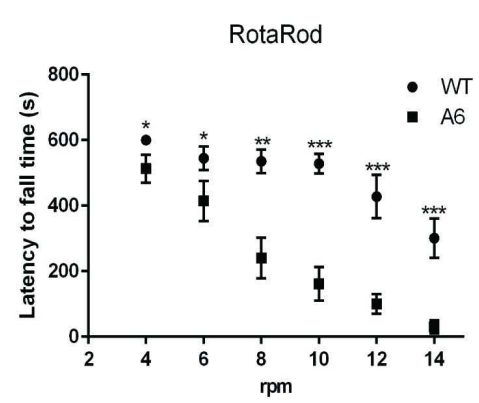

PERG

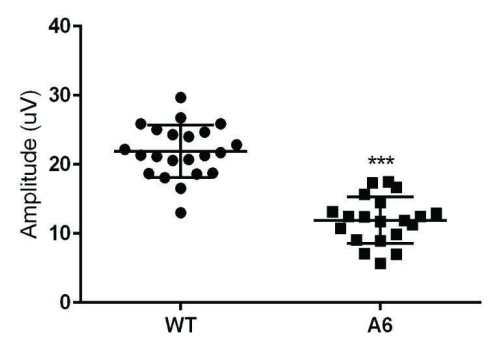

h

Body weight

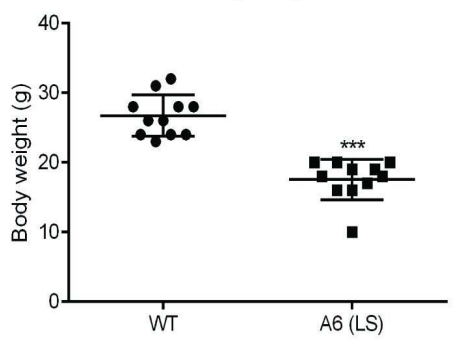

FERG

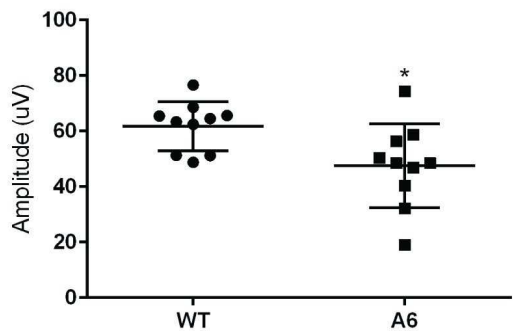



mutant human m.8993T $>$ G ATP6. a. Illustration of sc-HSP-mutATP6FLAG + mCherry plasmid containing the human mitochondrial heavy strand promoter (HSP) driving expression of the mutant human m.8993 T>G ATP6 subunit gene fused to FLAG with a stop codon followed by mitochondrial-encoded mCherry. Founder mutant ATP6 transgenic mice generated by microinjection of mito-targeted AAV2-sc-HSP-mutATP6FLAG + mCherry into blastocysts. b. Mutant human $\mathrm{m} .8993 \mathrm{~T}>\mathrm{G}$ ATP6FLAG + mCherry detected by confocal laser scanning ophthalmoscopy (CLSO) of retinas of live A6 founder mice; infrared focused on the inner retina (left panel) and in fluorescence mode (right panel) (arrows indicate fluorescent retinal cells). c. Founder A6 female crossed with wild type (WT) CB57BL/6 males. d. Cumulative proportional survival curve of A6 mice up to 6 months after birth for each generation ( $\mathrm{n}=101, p<0.0001$, log-rank test). e. Transgenic A6 mouse with hind limb paralysis $(\mathrm{n}=16$, see Supplementary Video 1). f. Transgenic A6 mouse with seizures ( $\mathrm{n}=4$, Supplementary Video 2). g. Latency-to-fall time of A6 founder mice decreased with increasing speed (4 to $14 \mathrm{rpm})$ compared to wild types ( $\mathrm{n}=12,{ }^{*} p<0.05$, ${ }^{* *} p<0.01$ and ${ }^{* * *} p<0.001$ ). h. Body weights of A6 mice with symptoms of LS (premature death) are significantly less than wild type mice at $\leq 6$-month-old $(\mathrm{n}=11, * * * p<0.001)$. i. Pattern electroretinograms (PERG) illustrate significantly decreased amplitude of A6 founder mice compared with wild types at age 7 months $(\mathrm{n}=10, * * * p<0.001)$. Statistical significance analysis of survival rates was performed by log-rank test for $\mathrm{d}$.

Basal ganglia lesions, retinopathy, and central nervous system neuronal degeneration of A6 mice. To determine whether A6 mice develop lesions of the basal ganglia, a condition seen in many human cases of $\mathrm{MILS}^{22}$, we imaged five A6 and one wild type brains by MRI. In one four-month-old, female F2 A6 mouse with quadriparesis and severe weight loss (Fig.2c \& Supplementary Video 3), we observed an increased T2

167 signal at the head of the caudate/putamen relative to the wild type, was bilateral and symmetric (Fig.2d168 2e). By the same MRI technique, the other 4 mice with only mild symptomatic LS disease were similar to 169 controls (Fig.2a-2b). Because of the high mortality rate observed in A6 mice, postmortem histological 170 examination was primarily used to characterize anatomical pathologies. Ocular histopathology of deceased 171 A6 mice revealed some retinas with normal ganglion cell, inner nuclear and outer nuclear layers (Fig. 2f) 172 and some with complete loss of the photoreceptor and outer nuclear layers (Fig. 2g, Supplementary Fig1c). 
173 Loss of the retinal photoreceptor layers is characteristic of retinitis pigmentosa (RP) of human NARP and

174 MILS. Light microscopy of A6 mouse brains revealed foci of spongiform encephalopathy where neurons

175 were lost, also a characteristic of human MILS (Fig. 2i-2j, Supplementary Fig. 1d) ${ }^{23}$. Transmission electron

176 microscopy of A6 mouse brains revealed, in addition to normal deep white matter with intact axons and

177 myelin (Fig. 2m\&n), clear cystic spaces at the lesion foci where fibers and neurons were decreased about

178 2-fold relative to control brains (Fig.2r). Examination of the cerebral cortices revealed increased prevalence

179 of electron dense structures in neuronal mitochondria that coexisted with normal mitochondria, reminiscent

180 of the mitochondrial degeneration that has been reported in MILS necropsy specimens ${ }^{24,25}$ (Fig. 2o-2q).

181 Abnormal cristae of neuronal mitochondria were also apparent with cross-sectional areas up to 2-fold wilder

182 than those of adjacent mitochondria (Fig 2s), a finding reported to be caused by loss of dimerization of the

183 ATP synthase ${ }^{25}$. 


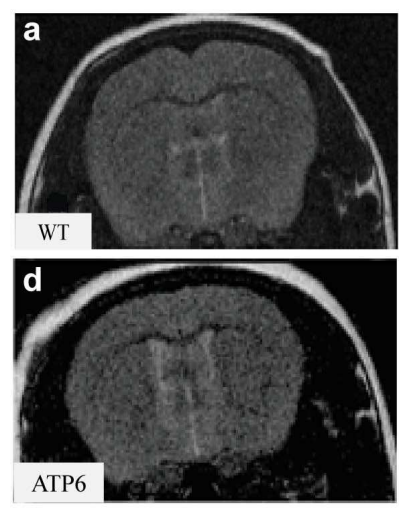

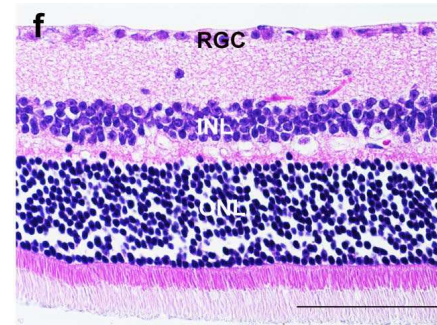
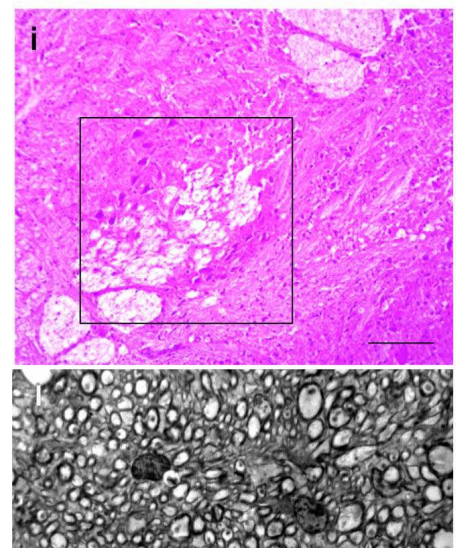

rosiso

56
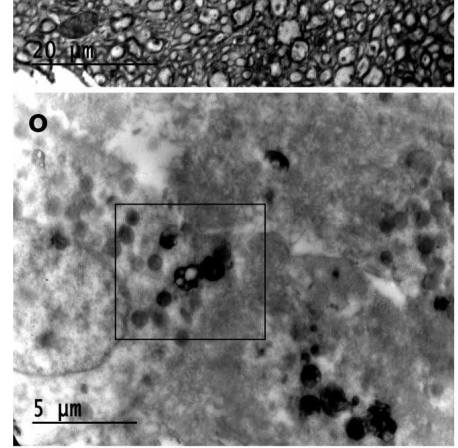

$r$

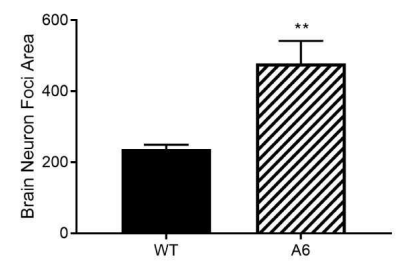

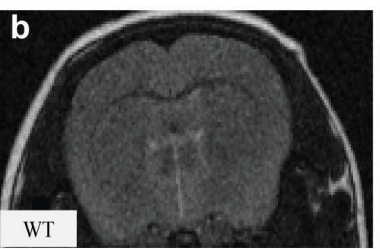
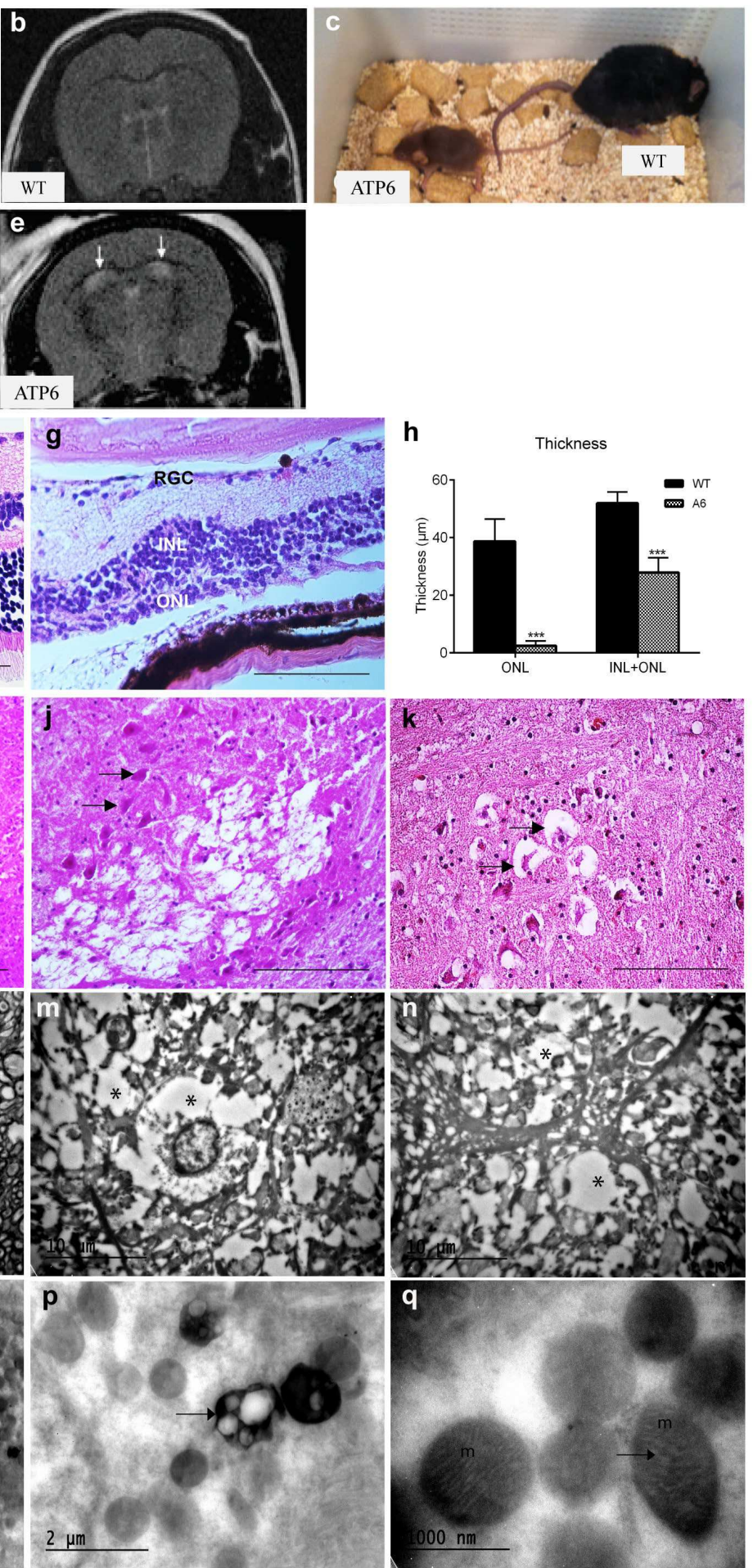

s

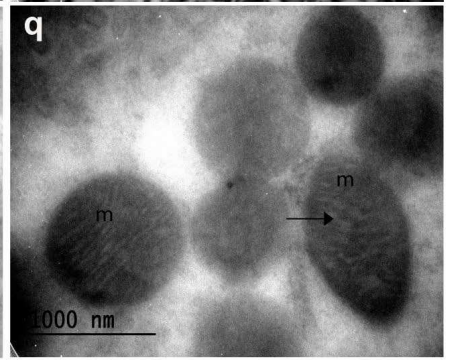

The width of Crista

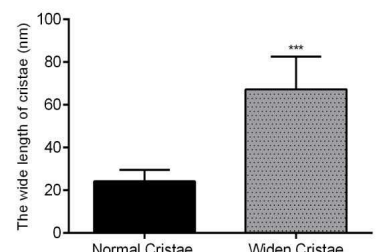


Figure 2 Brain MRI imaging and histopathology of A6 mice. a-b. T2-weighted MRI imaging of wild type mouse brain. c. Images of 4-month-old female quadriplegic A6 (F2) and wile type mice used for MRI imaging. d-e. T2weighted MRI imaging of the brain of A6 mouse shown in Fig. c (see also Supplementary Video 3) showing a bilateral and symmetric T2 signal increase at the head of the caudate/putamen (arrows), relative to wild type (a-b). f. Histopathology of an A6 mouse eye shows a normal-appearing retinal ganglion cell (RGC) layer, inner nuclear layer (INL) and outer nuclear layer (ONL) (scale bar: $20 \mu \mathrm{m}$ ). g. Histopathology of a different A6 mouse eye shows only the ganglion cell and inner nuclear layers with loss of the outer nuclear layers (scale bar: $20 \mu \mathrm{m}$ ). $\mathbf{h}$. Quantification of the ONL of A6 mice reveals significant loss relative to wild type $\left(\mathrm{n}=6,{ }^{* * *} \mathrm{p}<0.001\right)$. i. Histopathology of the A6 mouse brain motor cortex exhibits spongiform encephalopathy with vacuolization and loss of neurons (scale bar: $20 \mu \mathrm{m}$ ). j. High magnification of the boxed area in panel (i) shows remaining neurons (arrows) and spongiform degeneration where neurons are lost (scale bar: $25 \mu \mathrm{m}$ ). k. Histopathology of A6 mouse brain exhibits encephalopathy with vacuolization and loss of neurons $(\mathrm{n}=10)$. 1 . Transmission electron microscopy of the subcortical A6 white matter shows normal myelinated fibers. m-n. At the foci of spongiform encephalopathy, neurons and axons are lost leaving vacuolated spaces $(*)$ and degenerating fibers (arrow). o. A6 neurons showing mitochondria with electron dense aggregates adjacent to normal mitochondria of the same cell. p. Boxed area of panel (o), showing mitochondria with electron dense aggregates (arrows). q. A6 mitochondria with widening of cristae (arrow). r. Quantification of the foci in A6 mouse brain shows a 2-fold increase relative to normal brain ( $\left.\mathrm{n}=3,{ }^{* *} p<0.01\right)$. s. Quantification reveals 2-fold wider cristae in affected areas of A6 neuronal mitochondria relative to adjacent normal cristae $(* * * p<0.001)$.

Heteroplasmy of mutant human m.8993T>G ATP6 in A6 mice. Next, we characterized expression of the human mutant ATP6 gene in mouse mitochondria of A6 founder and offspring mice. PCR analysis of DNA extracted from transgenic A6 mouse brain, spinal cord or liver mitochondria using human ATP6 specific primers revealed the presence of the mutant human ATP6 gene (Supplementary Fig.1a\&b). Sanger DNA sequencing confirmed that the PCR fragments were mutant human m.8993T $>$ G ATP6 (Supplementary Fig.1c). SDS-PAGE western blot analysis showed that A6 mouse mitochondrial proteins reacted with FLAG and/or mCherry antibodies (Supplementary Fig. 1d). Immunofluorescence imaging of mutant human ATP6 fused FLAG revealed a perinuclear pattern in A6 mouse brain and spinal cord neuronal cells indicative of mitochondrial localization (Supplementary Fig. 1e). Then, we examined the ratio of 
mutant human ATP6 DNA relative to the endogenous mouse allele from whole tissues by semi-qPCR and

221 quantitative real time (q-rt) PCR. The data indicate low ratios of human ATP6 to MsATP6 (Supplementary

222 Fig.2a-c). Such ratios declined significantly in brains from subsequent F3 to F6 progeny (Supplementary

223 Fig. 3c). Laser capture microscopy of founder mouse brain and retinal layers followed by qPCR revealed

224 that human ATP6 levels were close to $10 \%$ in some areas of the brain, and up to $40 \%$ in the retinal outer

225 layer (Fig. 3a \& Supplementary Fig. 3d). By applying similar methods of quantification, the corresponding

226 ratios from F1 early death brains ranged from 10\%-20\% (Fig. 3b), while some of those from F2 and/or later

227 generation of mice with severe LS symptoms were as high as 70\% (Supplementary Fig. 3e). Although

228 higher mutant loads have been described in MILS and NARP autopsy specimens, low mutant loads have

229 also been associated with shortened lifespan phenotypes ${ }^{26-28}$, consistent with proposals that factors in

230 addition to overall mutant mitochondrial DNA load play important roles in determining disease

231 phenotypes ${ }^{29}$. In addition, based on the uneven distribution of mCherry fluorescence described above, it

232 seems probable that the mutant ATP6 DNA loads are dependent on the anatomical location of the affected

233 tissues, particularly neurons (Supplementary Fig. 2f upper) and may, for example be influenced by the high

234 bioenergetic requirements and larger mitochondrial densities of neuronal tissues.

\section{Mutant human m. 8993T>G ATP6 protein assembles into murine complex $V$ and impairs synthesis}

and hydrolysis of ATP. High magnification immunostaining revealed that the transgenic mutant human

237 ATP6-fused flag protein complex co-localized with the mitochondrial marker Porin in A6 mouse brain and

238 spinal cord mitochondria (Fig.3c). This finding supports the expression of mutant human ATP6 in murine

239 mitochondria. To determine whether mutant human ATP6 protein assemble into the murine complex V, we

240 performed one and two-dimensional blue native PAGE western blots. One dimensional blot probed with an

241 anti-FLAG antibody identified a band corresponding to the FLAG-mutant human ATP6 fusion protein that

242 migrated with complex V (Fig 3d, upper panel). Two-dimensional native blue/SDS-PAGE western blots

243 also probed with anti-FLAG and anti-mCherry with a different blot, identified $\sim 60 \mathrm{kDa}$ bands that represent

244 ATP6FLAG-mCherry (MW 58 kDa, Fig.3d-1\&2). We then reacted another gel with anti-ATP5a and 
confirmed its reactivity with the $\sim 60 \mathrm{kDa}$ band (MW. $\sim 55 \mathrm{kDa}$ ), the band identified as ATP6FLAG246 mCherry, consistent with the migration of complex V (Fig.3d-3). With increased denaturing time the small 247 band ATP6FLAG was decreased or lost, consistent with our results from SDS-PAGE western blots which 248 only show the $\sim 60 \mathrm{kDa}$, ATP6FLAG-meCherry band (Supplementary Fig. 2d). A possible explanation for 249 a fusion protein is that the stop codon between ATP6FLAG and mCherry allow read through. To confirm 250 the identity of complex V in western blots, the anti-FLAG antibody reactive bands were excised from one 251 dimensional blue native gel, digested with trypsin to prevent the release of membrane bound proteins ${ }^{24}$ and 252 analyzed by mass spectroscopy. The results confirmed identity of the ATP synthase and therefore the 253 assembly of ATP6FLAG into complex V (Supplementary Fig. 4c \& Table 6). To investigate the effects of 254 mutant human ATP6 on complex V function, we implemented in gel complex V activity assays. As shown 255 in Fig. 3e and Supplementary Fig. 4a, we found that ATPase activity was impaired in parallel with the lost 256 dimer bands from A6 mouse brain, spinal cord and muscle ATPase. In addition, the rate of ATP synthesis 257 and oxidative phosphorylation (OXPHOS) in A6 brain extracts was decreased significantly, by $\sim 50 \%$ 258 compared to wild type brains (Fig. 3f). Taken together, these findings are consistent with assembly of 259 mutant human A6 subunits into the murine complex V with corresponding loss of complex V activity, 260 OXPHOS and mitochondrial ATP generation in brain, spinal cord and muscle of A6 mice. 
a

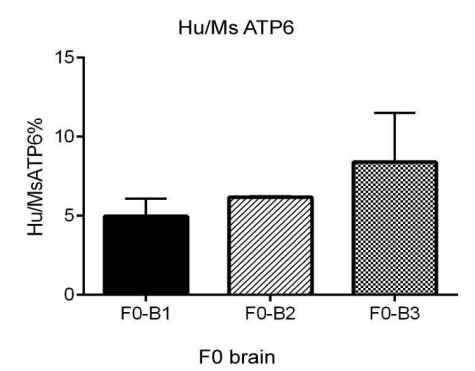

b

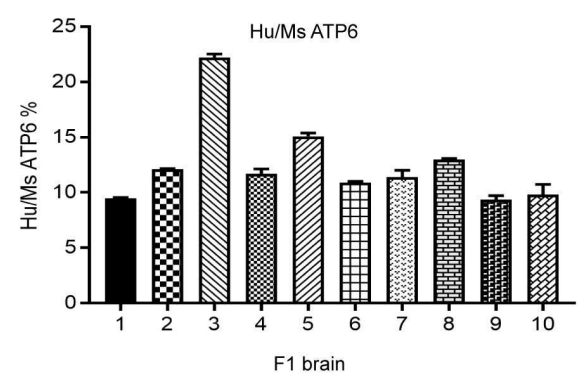

C
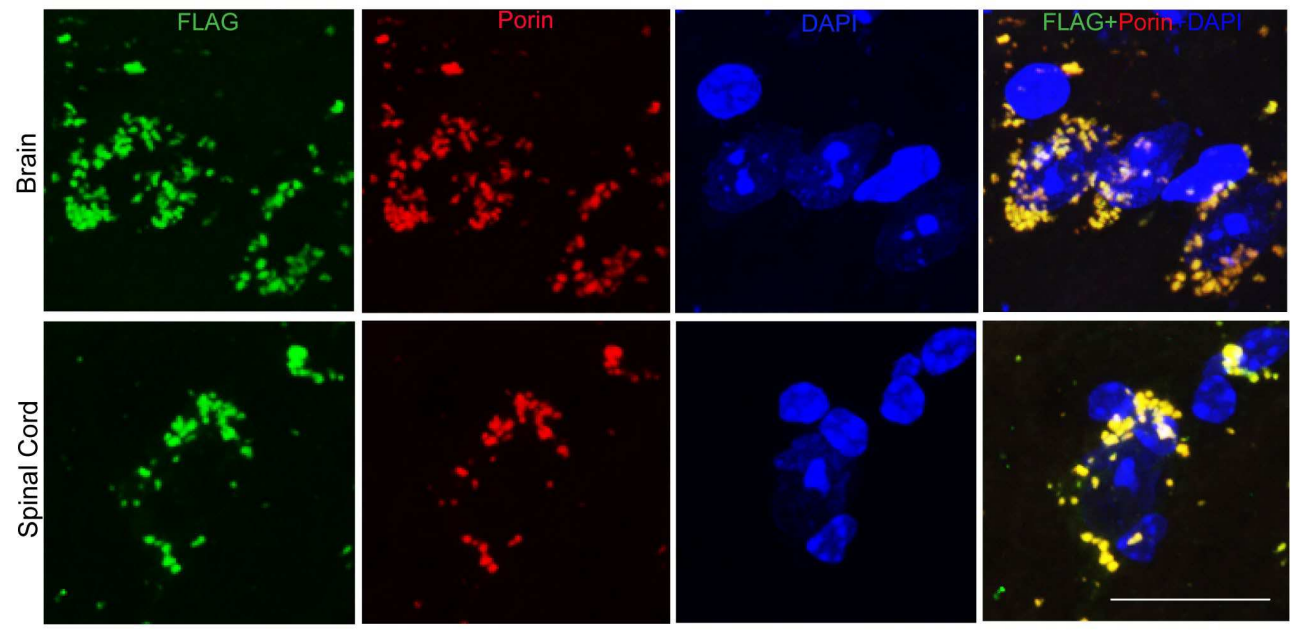

d

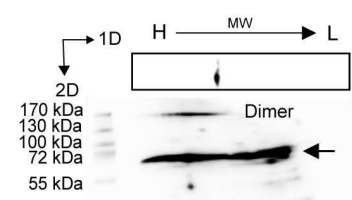

FLAG

$36 \mathrm{kDa}$

e

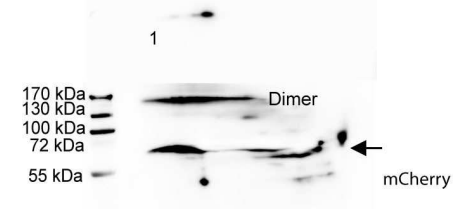

$36 \mathrm{kDa}$
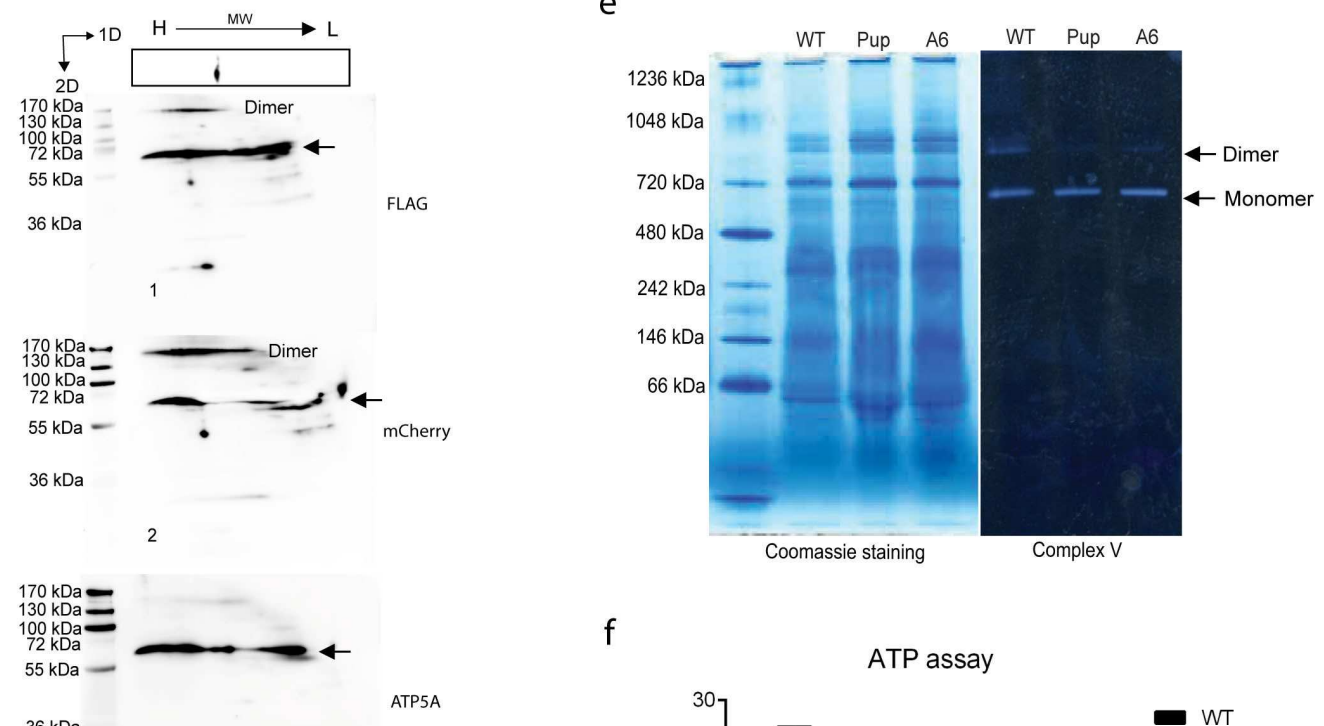

f

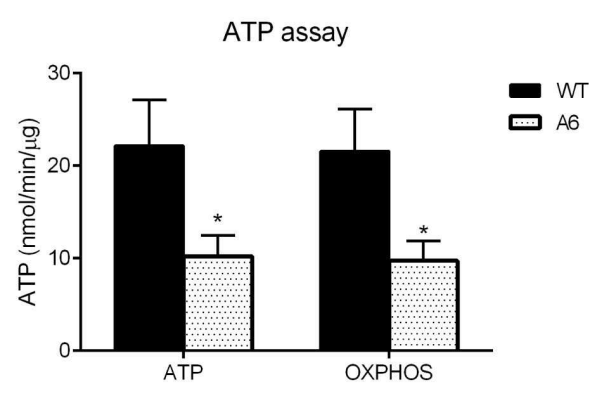


Fig 3. Reduction of complex $V$ activity and ATP synthesis by mutant human m.8893T $>$ G ATP6. a. Quantitative rtPCR after laser capture microdissection of positive mCherry cells showing $<10 \%$ ratio of mutant human ATP6 to mouse endogenous ATP6 in founder A6 mouse brains. b. Quantitative rtPCR showing ratios of mutant human ATP6 to mouse endogenous ATP6 of $10-20 \%$ in F1 premature death pup brains. c. High magnification immunostaining showing mutant human ATP6 fused flag co-localized with mitochondrial marker Porin in A6 mouse brain and spinal cord mitochondria (scale bar: $50 \mu \mathrm{m}$ ). d. Two-dimensional native blue/SDS-PAGE western blots probed with 272 antibodies against Flag and mCherry indicate migration of ATP6FLAG + mCherry $(\sim 60 \mathrm{kDa})$ consistent with complex $273 \mathrm{~V}$, and co-migrating at a similar molecular size as ATP5A ( $\sim 60 \mathrm{kDa})(\mathrm{n}=3$, independent experiments). e. Complex V in gel activity assay showing decreased intensity of complex V dimers in A6 mouse brain relative to wily type brain $(\mathrm{n}=10)$. f. ATP synthesis and OXPHOS in A6 mouse brains were decreased by $>50 \%$ relative to wild types $(\mathrm{n}=5$, $* p<0.05)$.

Construction of mito-targeted AAV9 with a cox8 signal sequence. To investigate the feasibility of a gene therapy strategy to ameliorate or preempt the disease phenotypes in this A6 mouse model of human MILS/NARP, we constructed a rescue vector by replacing the mutant human ATP6 with wild-type human ATP6 (Fig. 4a). Unlike AAV2, AAV9 efficiently crosses the blood-brain barrier following intravenous injection therefore we used AAV9 serotype for the rescue ${ }^{30}$. A cox 8 signal sequencing was added to the $\mathrm{N}$ terminal of the VP2 sequence to target wild type human ATP6 to mitochondria. Western blots of the packaged mito-targeted AAV9 vector stained respectively with anti-COX8 and AAV antibodies confirmed a product with the predicted slower mobility relative to the native VP2 (Fig. 4b, left and right panel). wild type ATP6. The presence of both mutant and wild type human ATP6 DNA in AAV9-transduced homoplasmic mutant m.8993T $>$ G ATP6 cybrids (NARP cells) were confirmed by amplifying DNA from transduced and non-transduced cells and digesting fragments with Sma I after 5-days selection in galactose media. The relative representation of wild type and mutant ATP6 can be determined because wild-type ATP6 lacks a Sma I site. As shown in supplementary Fig. 5a, whereas DNA from non-transduced cybrids was completely digested by Sma I, the fragments from AAV9-transduced cells were incompletely digested. 
The identity of the undigested band was confirmed by excision from the gel and digestion with BstN1 that cuts wild-type ATP6, but not mutant human ATP6 (Supplementary Fig. 5a, lane 4). Using Sanger DNA

294 sequencing, we further confirmed that the PCR fragments derived from transduced cells contained both 295 mutant and wild-type human ATP6 alleles (Supplementary Fig. 5b, upper), whereas the amplificants 296 obtained from uninfected cells had only the $\mathrm{T}>\mathrm{G}$ allele (Supplementary Fig. 5b, bottom). The ratio of wild 297 type to mutant ATP6 in mito-targeted AAV9 transduced cybrids, determined by relative band intensities was $25-47 \%$. Immunofluorescence microscopy of transduced cybrids after staining for FLAG (present as ATP6FLAG in the AAV9 rescue vector) revealed perinuclear FLAG expression that co-localized with MitoTracker Red (Fig.4c). As a control, immunofluorescent images of homoplasmic m.8993T $>$ G cybrid cells transduced with AAV9 containing wild-type ATP6FLAG without the cox8 targeting signal showed ATP6FLAG expression confined to the nucleus (Supplementary Fig.5e). Transmission electron microscopy of cybrids at 4 hours after infection with mito-targeted AAV9 revealed viral particles associated with membrane-bound organelles (Fig.4d-4f, Supplementary Fig.5c). One-dimensional blue native/PAGE western blots of mitochondria from transduced cybrids confirmed reactivity of a $550 \mathrm{kDa}$ holo-complex V comparing uninfected and transduced cybrids showed monomer and dimer bands with transduced cybrid cells with stronger intensities of assembled dimers. (Fig.4h). Together, these findings confirm successful transfer with subsequent expression and activity of wild type human ATP6 by mito-targeted AAV9 in the mitochondria of homoplasmic m.8993T $>$ G cybrid cells.

\section{Rescue of homoplasmic mutant m.8993T $>$ G ATP6 cybrids by mito-targeted wild type ATP6.}

312 Transduction of homoplasmic mutant m.8993T $>$ G ATP6 cybrids with mito-targeted wild type ATP6313 AAV9 significantly increased the viability of cells cultured in glucose-free, galactose media (selection 314 media) relative to uninfected cybrids (Fig. 4i). Luciferin-luciferase assays to measure ATP with malate and 315 pyruvate substrates, revealed significantly increased rates of ATP synthesis of 2.82, 3.49 and 3.01 $316 \mathrm{nmol} / \mathrm{min} / \mu \mathrm{g}$ (Fig. 4j) in cybrids transduced respectively at MOIs of 500, 1,000 and 5,000, compared with 
$3171.3 \mathrm{nmol} / \mathrm{min} / \mu \mathrm{g}$ of uninfected cybrids. Accordingly, the derived rate of OXPHOS was also significantly 318 increased, respectively to $2.44,2.79$ and $2.7 \mathrm{nmol} / \mathrm{min} / \mu \mathrm{g}$ at MOIs of 500, 1,000 and 5,000, relative to 0.93 $319 \mathrm{nmol} / \mathrm{min} / \mu \mathrm{g}$ of uninfected cybrids (Fig. $4 \mathrm{j})$

320

321

322

323

324

325

326

327

328

329

330

331 
a
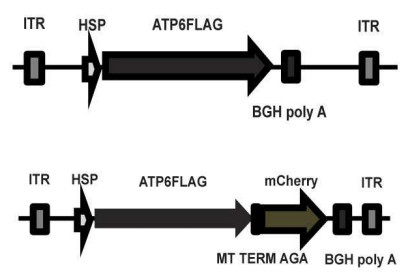

C
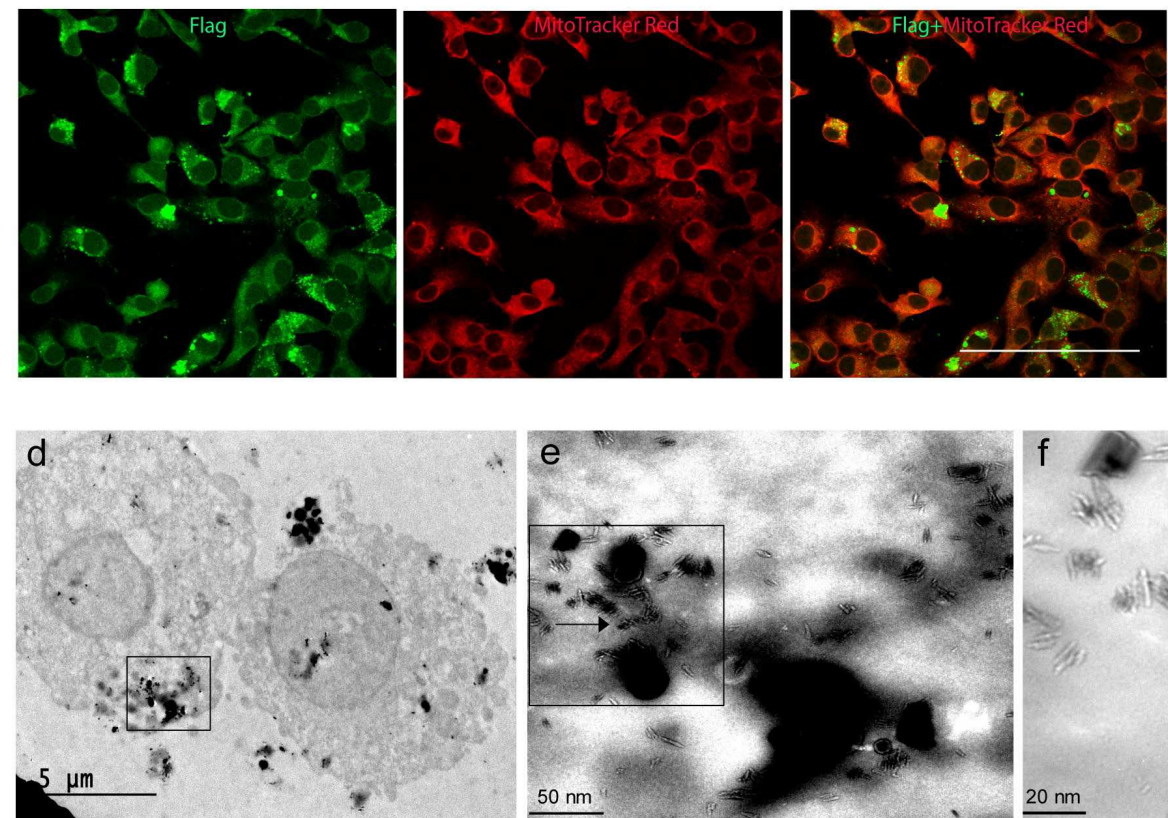

g

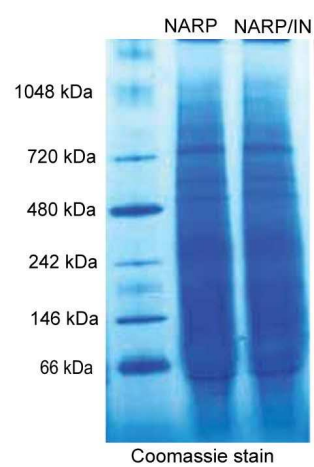

Flag

i

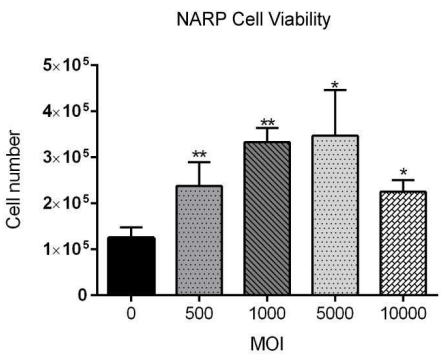

b

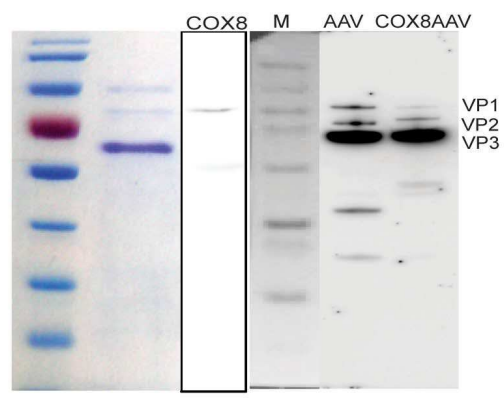

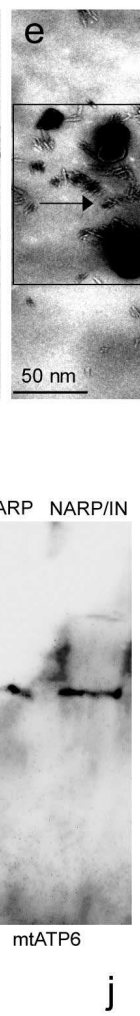

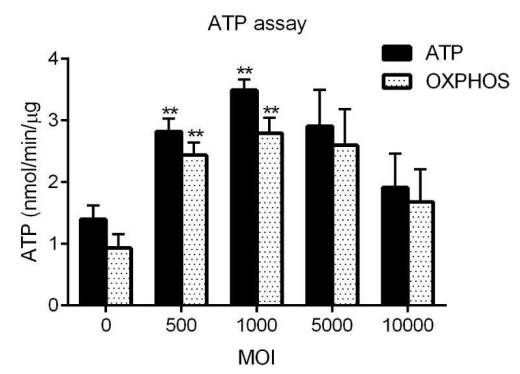

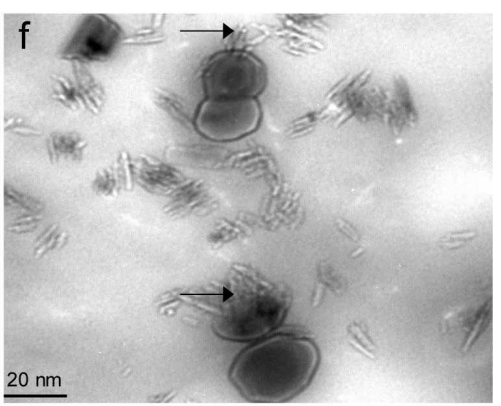

h

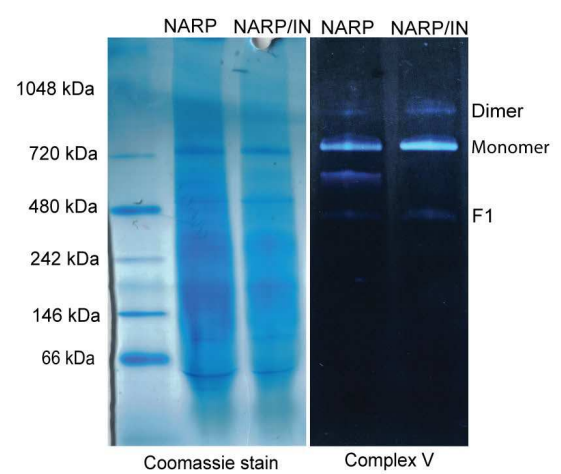



ATP6. a. Illustration of the sc-HSP-ATP6FLAG plasmid (upper panel) containing the human mitochondrial heavy strand promoter (HSP) driving expression of wild-type human ATP6 fused in frame to a FLAG epitope and the scHSP-ATP6FLAG-mCherry plasmid (bottom panel) that contains mitochondrially encoded mCherry. b. Western blot of mitochondria, COX8-targeted AAV9 stained with anti-COX 8 antibody (left panel). Western blot showing that VP2-COX8 is larger than the unmodified VP2 (right panel). c. Cybrids infected with mito-targeted AAV9 containing wild-type ATP6FLAG selected in glucose-free galactose media exhibit perinuclear FLAG expression that co-localizes with MitoTracker Red (bottom panels, scale bar: $20 \mu \mathrm{m}$ ). d. Transmission electron microscopy of cybrids transduced with mito-targeted AAV9 display mito-targeted AAV viral particles compartmentalized in membrane bound vesicles. e-f. Higher power images reveal that the contents of the viral particles are selectively delivered to mitochondria (arrows) rather than the nucleus $(\mathrm{Nu})$. g. One dimensional native blue/PAGE (left panel) western blot showing a band from infected cybrids (NARP/IN) detected by anti-FLAG antibody stain (middle panel) also reacted with a mt-ATP6 antibody confirming its identity as ATP6 (right panel). h. In gel complex V activity assay showing increased intensities of dimer and monomer bands of complex $\mathrm{V}$ in infected (NARP/IN) versus uninfected cybrids (NARP). $\mathbf{i}$. Survival of AAV9 transduced cybrid in galactose selection media was significantly and dose-dependently increased relative to uninfected cybrid cells at MOIs of 500 and $1,000\left({ }^{* *} p<0.01,{ }^{*} p<0.05\right)$. j. Relative to uninfected cybrids, the overall rate of ATP synthesis and OXPHOS of infected cells increased significantly and dose-dependently with MOIs of 500 and $1,000\left({ }^{* *} p<0.01\right)$. Data are representative of three independent experiments with similar results.

Expression of wild type human ATP6 in mouse brain following intravenous delivery of mito-targeted

AAV9. Because AAV9 crosses the blood-brain barrier and has been reported to rescue mice with LS caused by a nuclear gene knockout ${ }^{8}$, we tested AAV9 transduction first in control mice by intravenously injecting $35410 \mu \mathrm{L}$ of self-complementary mito-targeted AAV9 $\left(1.86 \times 10^{12} \mathrm{vg} / \mathrm{mL}\right)$ expressing wild-type human ATP6FLAG+mCherry into the right orbital venous sinus. Using laser-scanning ophthalmoscopy focused on the inner retina (Fig. 5a), we detected ATP6FLAG+mCherry protein in both right (Fig. 5b) and left eyes (Fig. 5c) of live injected mice but not in noninjected control mice. Relative to noninjected mice (Fig. 5e-h), confocal microscopy of the brains of injected mice further revealed fluorescence of the 
360 Punctate and perinuclear ATP6FLAG+mCherry stained by FLAG colocalized with porin, a mitochondrial 361 marker (arrow shown in Fig. 5n of Fig. 5m-5p and at higher magnification in Fig. 5r of Fig. 5q-5u). These 362 findings suggest that the AAV9 vector traversed the blood-brain barrier into mitochondria of central 363 nervous system (CNS) neurons. Therefore, we tested whether delivery of our AAV9 vector was effective 364 in preventing and/or reversing symptoms of MILS in this mouse model.

365

366

367

368

369

370

371

372

373

374

375

376

377

378

379

380

381 
a
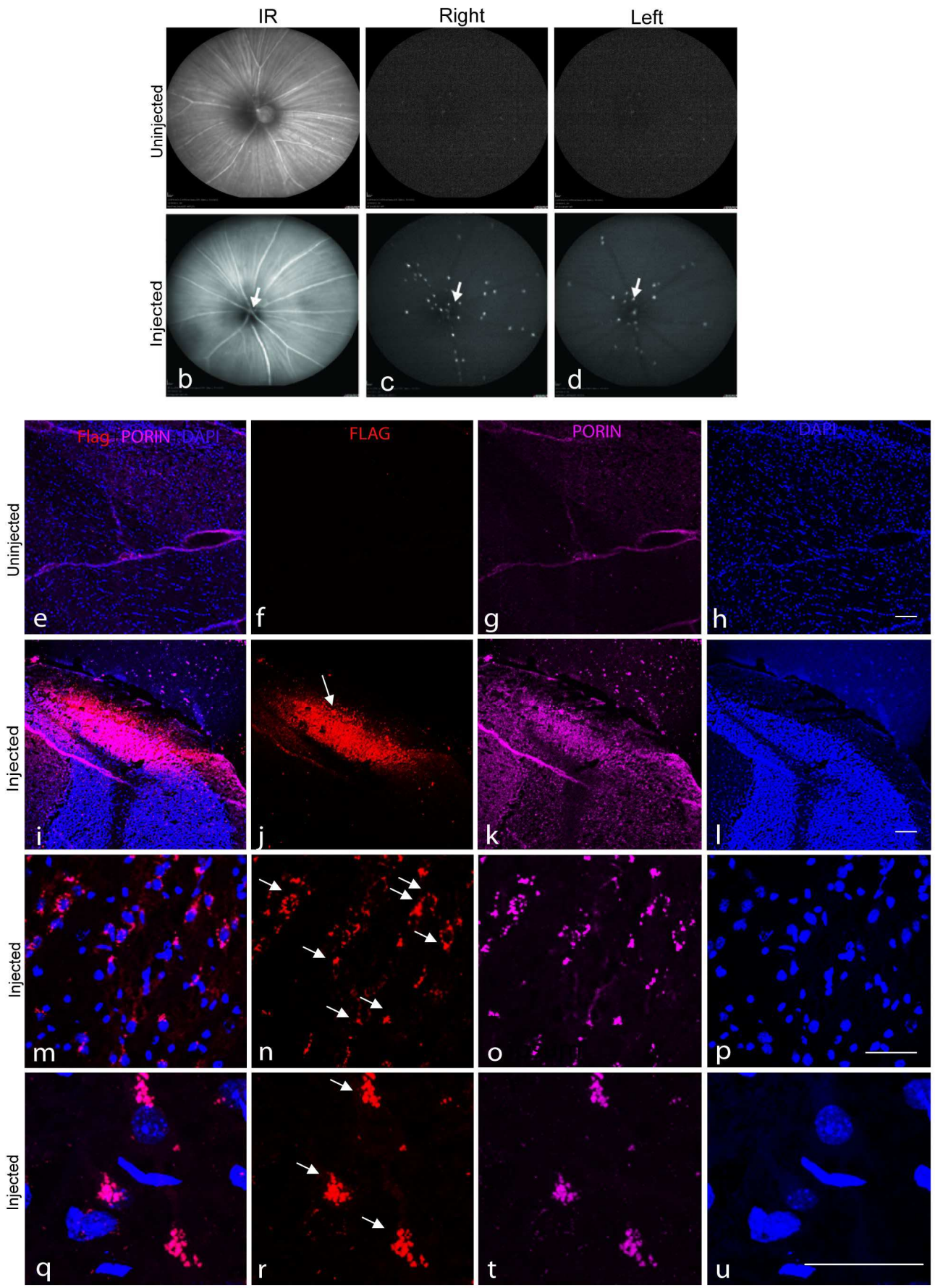

383 Figure 5 Intravenous administration of mito-targeted AAV9 with wild-type human $A T P 6$ to wild type mice. a.

384 Illustration of intravenous injection into the mouse orbital venous sinus $(\mathrm{n}=7)$. b. Laser scanning ophthalmoscopy of 385 a live mouse focused on the inner retina (infrared=IR) with mCherry labeled retinal cells in the right (c) and left eyes (d) (arrows indicate the optic nerve head). e-h. Postmortem micrographs of an untreated mouse brain reveal absence 
of FLAG immunostaining. i-l. Micrographs of injected mouse brains show FLAG tagged ATP6 in the motor cortex (arrows) (Scale bar: $50 \mu \mathrm{m}$ ). m-p. and q-u. Higher magnification shows punctate and perinuclear ATP6FLAG that colocalizes with mitochondrial porin (arrows, Scale bar: $20 \mu \mathrm{m}$ and $10 \mu \mathrm{m}$ ).

human ATP6. Transgenic $A 6$ mice that developed symptoms of severe paralysis or hunching and that were assigned as high risk for imminent death, were administered intravenous injections of $1.86 \times 10^{10} \mathrm{vg}$ of mitotargeted AAV9 containing wild-type human ATP6. Figure 6a shows examples of the locomotion of a highresponder rescued mouse that had severe hind limb paralysis prior to treatment. Symptoms appeared to stabilize at post injection $>$ Day 3, and the mouse survived to Day 65 (Supplementary Video 4). Rescues were initiated in two groups and survival and pathology were tracked subsequent to rescue injections (D0). Group 1 included similarly aged A6 mice with severe hind limb paralysis. Log rank tests showed a 28-day mean survival (range 5-65 days), of such mice in the treatment group ( $\mathrm{n}=11)$, while 5 untreated A6 mice with similar hind limb paralysis died within 4 days of D0 (Fig.6b). In group 2, that included similarly aged A6 mice with severe hunching, using the same protocol, the mean survival of treated mice $(n=14)$ was 16 days (range 5-47 days), compared with $<3$ days for the non-rescued hunching group ( $n=12$ ) (Fig. 6c). The results are consistent with the rapid expression time and phenotypic complementation by the scAAV9groups relative to non-rescued controls. 

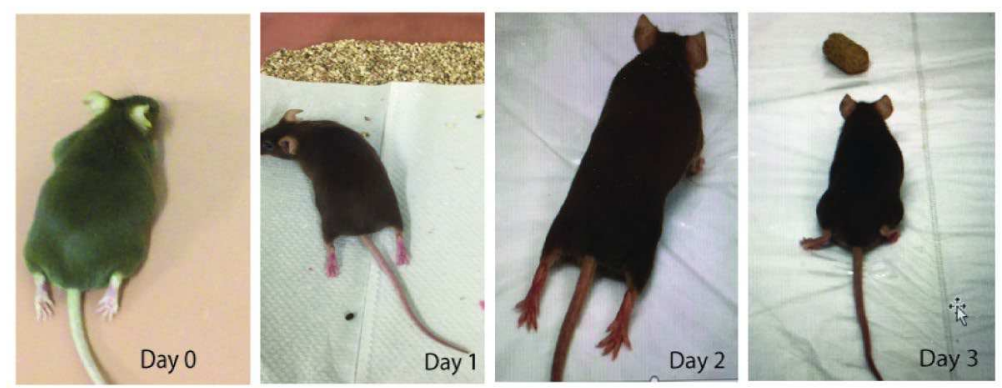

b
Surivival of Paralysis A6

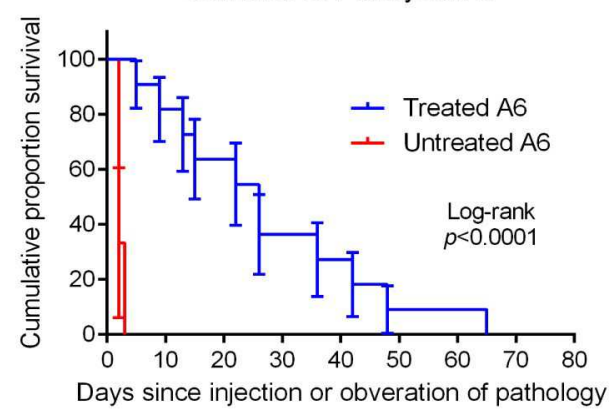

Survival of Hunching A6

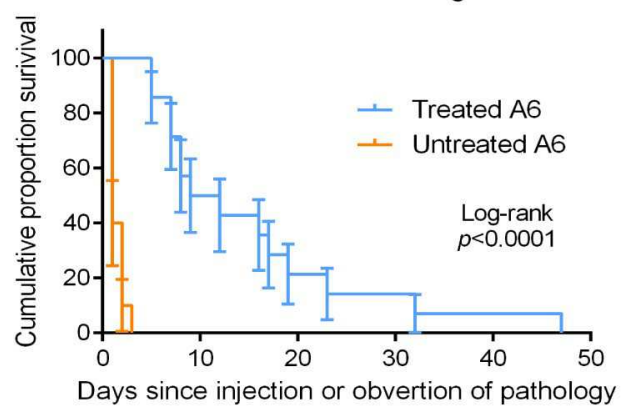

Fig. 6 Amelioration of A6 mouse LS disease onset by intravenous administration of AAV9 with mito-targeted wild type ATP6. a. A6 mouse with hind limb paralysis with mito-targeted AAV9 containing wild-type human ATP6 delivered by i.v. injection at D0. No change in paraplegia was seen during the subsequent 2 days whereas by day 3 , an improvement in hind limb function was apparent (see Supplementary Video 4). b. Log-rank test showing significantly extended lifespans of rescued paretic A6 mice compared with untreated controls $(\mathrm{n}=16, p<0.0001)$. c. Log-rank test showing significantly extended lifespans of hunched A6 mice relative to untreated hunched controls $(\mathrm{n}=26, p<0.0001)$.

Suppression of LS phenotypes and enhanced Complex V activity by early rescue. Preventative treatment was initiated in non-symptomatic 3-month F2 A6 mice that were selected from the progeny of high-mortality F1 dams. Thirty-four mice from 3 different A6 mice were divided randomly into 10 rescue and 24 non-rescue controls. The treatment group received intravenous injections of $1.86 \times 10^{10} \mathrm{vg}$ mitotargeted AAV9 rescue vector while controls received PBS. All rescue animals (100\%) survived through 21 months, while untreated A6 mice sustained 8 of 24 premature deaths between 5-12 months and a cumulative 
432 survival rate of $67 \%$ (Fig. 7a). Log rank tests after 15+ months, indicate 56\% (9 out of 16) of untreated A6

433 mice presented severe LS phenotypes including paralysis (1), hunching (4), weight loss (3) and tumors (1),

434 whereas $80 \%$ of the treatment group were non-symptomatic. The exceptions were 2 treatment mice that

435 showed significant weight loss (Fig. 7b). Three months after injection, visual function measured by pattern

436 ERG, was significantly improved in treated A6 mice over that of the untreated group (Fig.7c).

437 Histopathological analysis confirmed that the brains and spinal cords of rescued A6 mice (Fig. 7e\&h) were

438 similar to wild-type mice with markedly less vacuolization and cell loss (Fig. 7d\&g) compared to the severe

439 loss seen in the non-rescue group (Fig. 7f\&i). In addition, non-rescued survivors lost an average of $30 \%$

440 body weight at age 1-year $(n=25)$, whereas the mean weights of rescued mice were no different from age-

441 matched wild-type controls. The ratio of heart to body weight of hunching non-rescued A6 mice was almost

442 twice that of wild-type or rescued A6 mice. Cardiomegaly, a component of MILS, was also apparent only

443 in the non-rescued A6 hearts (Fig. 71). Immunofluorescence images of mutant human ATP6 fused FLAG

444 in heart sections revealed a perinuclear expression pattern consistent with presence of the mutant human

445 ATP6 protein in heart mitochondria (Supplementary Fig. 2f, bottom panel). In-gel activity measurements

446 of ATP hydrolysis to assess complex V activity revealed significantly improved ATPase activity of rescued

447 A6 mice (brain) relative to untreated A6 mice (Fig. 7m\&n). The results are consistent with efficient AAV9-

448 mediated delivery of wild type ATP6 by intravenous injection, complementation of the defective mutant

449 ATP6 and effective amelioration of the MILS phenotypes by early gene delivery in this model. 

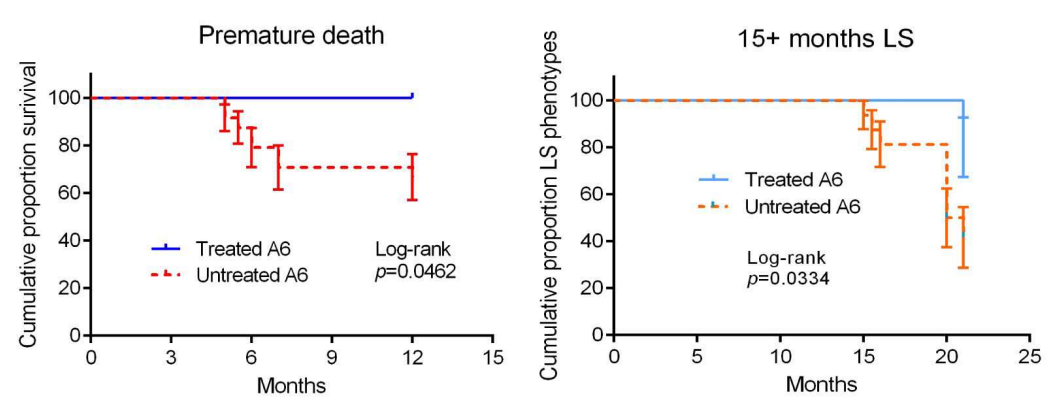

C
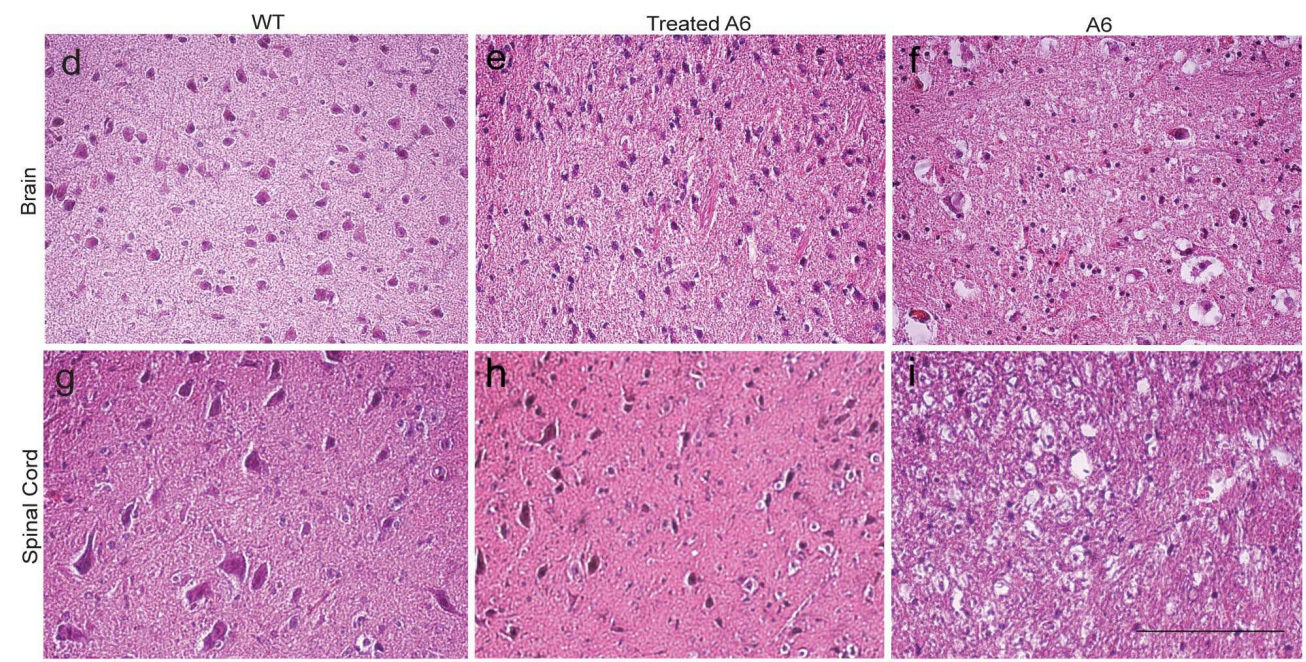

k

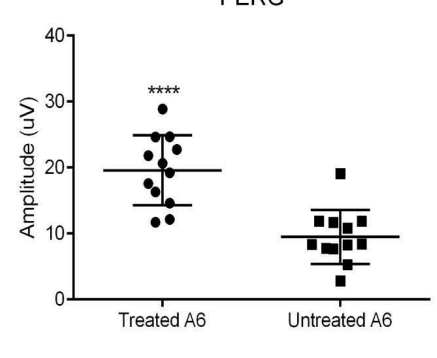

j
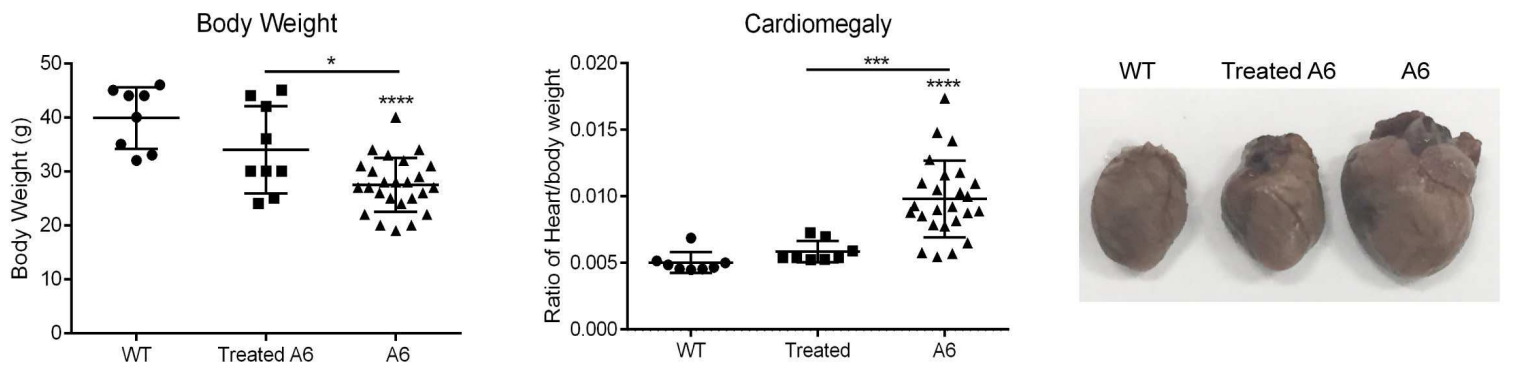

m

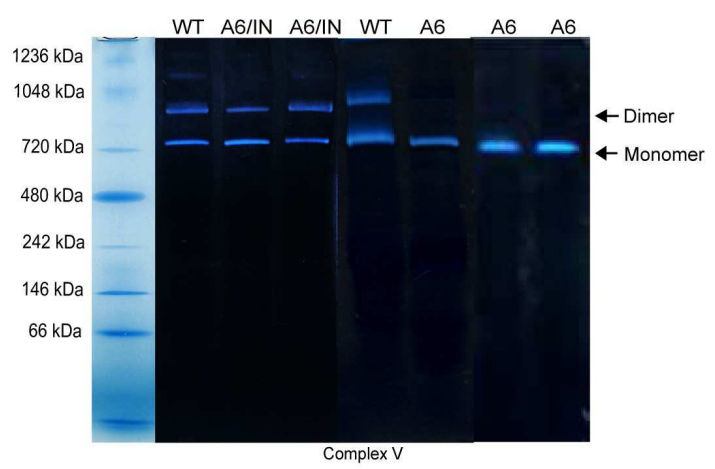

$n$

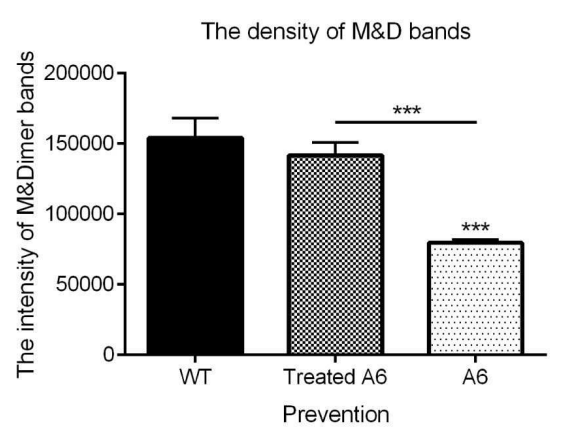

Prevention 

type $\boldsymbol{A T P 6}$. a. Cumulative proportion survival curves showing $100 \%$ survival of treated A6 mice through 12 months and untreated animals with a cumulative $67 \%$ Kaplan-Meier survival rate also at 12 months ( 8 of 24 died before 12 months, $\mathrm{p}=0.0462$, log-rank test). b. Log rank test showing untreated A6 mice had cumulative LS phenotypes of $56 \%$ (9/16) including paralysis (1), hunching (4), weight loss (3) and tumor (1) at age $15-21$ months, whereas $80 \%$ of rescued mice presented with normal (no disease) phenotypes at age 21 months, while the remaining 2 mice $(20 \%)$ showed weight loss as the only identified LS-related phenotype (Log-rank, $p=0.0217$ ). c. Pattern ERG analyses of treated mice showed an almost 2 -fold increase in amplitude relative to untreated controls $(\mathrm{p}<0.001)$. (d-f) Neuropathology respectively of wild type, rescued and non-rescued A6 mouse brains showing cystic spaces and neuronal loss only in the untreated group. (g-i) Neuropathology respectively of wild-type, treated and untreated mouse spinal cords showing cystic spaces and neuronal loss in untreated groups (scale bar: $20 \mu \mathrm{m}$ ). (j) Body weights of untreated A6 mice are significantly less than those of treated A6 mice and age-matched wild-types $\left({ }^{*} p<0.05\right.$, $\left.*_{* *}<0.001\right)$. k. Heart to body weight ratios of treated A6 mice are similar to those of wild-type mice with significantly less cardiomegaly than untreated A6 mice (wild-type vs A6, $* * * * p<0.0001$; treated A6 vs A6, ${ }^{* * *} p<0.001$ ). $\mathbf{l}$. Representative hearts of wild type (WT), treated A6, and untreated A6 mice with cardiomegaly. $\mathbf{m}$. In gel activity assays for complex V showing increased intensity of dimer F0F1 ATPase bands of treated A6 mouse brain prior to disease onset compared to untreated A6 mice. n. Quantification of the density of the monomer and dimer F0F1 ATPase bands of treated A6 mice prior to disease-onset (A6/IN) shows significantly increased dimer F0F1 ATPase bands relative to untreated A6 mice and no significant difference from wild types $(\mathrm{n}=3, * * * p<0.001)$.

470 Heteroplasmy of human ATP6 alleles in rescued A6 mice. We used PCR and restriction fragment length 471 polymorphism (RFLP) to quantify heteroplasmy of mitochondria in the $A 6$ rescue moue brain, spinal cord 472 or liver mitochondrial DNA. Sequencing of PCR amplified DNA obtained from mitochondria of rescued 473 A6 mice revealed the presence of both wild type and mutant human ATP6 alleles (Supplementary Fig. 6a\&b). To determine the relative contributions of wild type and m.8993T $>$ G mutation of ATP6, the signal intensities of the relevant DNA bands separated by agarose gels were measured using image $J$ and the corresponding percentages calculated for each sample. As shown in Supplementary Figure 6c, the ratio of

477 wild type to mutant ATP6 in rescued mice was close to $50 \%$. Western blots confirmed $\sim 10$-fold increased 
478 intensity of the ATP6FLAG+mCherry protein band in samples from rescued versus non-rescued A6 mice

479 from brains (Supplementary Fig. 6d\&e).

480

481

482

483

484

485

486

487

488

489

490

491

492

493

494

495

496

497

498

499

500

501

502

\section{Discussion}

We used a mito-targeted AAV2 vector microinjected into mouse blastocysts to mediate germline transmission of mitochondrial encoded subunit 6 of the human ATPase gene variant m.8993T $>$ G. Female founder mice with high ocular expression of the transgene were backcrossed with wild-type C57BL/6 males for 6 generations to generate a total of $53 \mathrm{~F} 0$ and 446 progeny $\mathrm{F} 1$ to $\mathrm{F} 6$ mice, $70 \%$ of which displayed one or more hallmark symptoms of LS/NARP. Presence of the m.8993T $>$ G variant DNA and protein in transgenic animals, localization to mitochondria and assembly into complex $\mathrm{V}$ with dysfunctional F1ATPase activity were confirmed by molecular, immunoblot and immunostain analyses of brain, spinal cord, ocular or liver mitochondria. Because of the prevalence of ocular defects and high vulnerability of neuronal ocular tissues to mitochondrial variants that cause LS including the m.8993T $>$ G mutation, we focused our molecular genetic analyses primarily on these tissues in F0, F1 and F2 mice. Combined laser capture and RT-qPCR analyses revealed variable heteroplasmy between different retinal locations that approached $40 \%$ in the outer nuclear layer and $20 \%-25 \%$ in the ganglion cell inner nuclear layers and around $10 \%$ in founder brain. This compared with 10\%-20\% in F1 brain sections. Representation of the m.8993T $>$ G variant decreased markedly in F3-F6 generations in favor of the wild type allele, and there was evidence of positive correlations between transgene expression and retinal cell loss. Sustained disease phenotypes of F1 and F2 generation mice despite the absence of significant transgene replication, may be attributed to our selection of high expression founder mice for F0 backcrossing, tissue-specific responses to mutation load, and the apparent absence of tight associations between disease phenotypes and degrees of heteroplasmy often seen in human mitochondrial disease (see below). The results are also consistent with previous studies from our group where we reported similarly variable tissue heteroplasmy after germ line delivery of a human ND4 m11778G >A gene variant to mouse mitochondria. In these studies, we reported a maximum transgene expression of $20 \%$ in the retinal RGC layer, $0.08 \%$ in the inner nuclear layer 
and undetectable expression in the outer nuclear layer. Mice with the variant mitochondrial gene displayed

504 a robust disease phenotype that closely mimicked symptoms of human LHON disease despite the relatively

505 low transgene penetration and limited replication ${ }^{9}$.

506 LS-related symptoms observed in 70\% of F0-F6 mice included premature death, paralysis, seizures, weight 507 loss, off balance and visual loss. Inclusive of all offspring, 32\% of mice died before 15 months of age with 508 F1 mice showing the highest mortality rate of 50\% at 6-months, followed by $23 \%$ mortality of F2 mice at 5096 months. Of surviving mice, $\sim 50 \%$ became hunched, paralyzed, developed visual loss and/or seizures and 510 weight loss with varying severity and age of onset. Together the results indicate widespread and pronounced 511 but variable disease phenotypes of A6 mice, in many respects similar to human MILS. The presence of the 512 most severe disease phenotypes in F0 and F1 mice and loss or reduced severity and prevalence of such 513 phenotypes in subsequent generations in parallel with decline of mutant gene loads is consistent with the 514 expected role of gene load in disabling mitochondrial functions and promoting disease expression in this 515 model.

516 The importance of gene load has also been described in patients with MILS/NARP, and whereas there is a 517 general association between degree of heteroplasmy and phenotype severity, precise correlations of clinical 518 phenotype with mutation load are rare with few clear thresholds corresponding to a given phenotype, and 519 many instances where severe symptoms are associated with lower mutant gene loads and vice versa ${ }^{1,31,32}$. 520 For example, Stendel et $\mathrm{al}^{33}$, recently quantified heteroplasmy and clinical presentation of 132 subjects with 521 known pathogenic MT-ATP6 mutations and related the genotype with neurological manifestation of the 522 disease. They reported high degrees of heteroplasmy but wide variations in both symptomatic and non523 symptomatic individuals. For symptomatic subjects, the mean heteroplasmy was $95 \%$ with a range of $20 \%-$ $524100 \%$, while for unaffected individuals the mean was $73 \%$, also with a range of $20 \%-100 \%$. Similarly, 525 while most cases of LHON are homoplastic, severe symptoms have been documented with as little as $33 \%$ 526 mutated mitochondrial $\mathrm{DNA}^{34}$, and a m.5545C $>$ T mutation affecting tRNA specificity that usually carries 527 a 70-90\% threshold was reported in one case study to confer a severe disease phenotype at less than $25 \%$ 
528 in affected tissues, and between 4 and $8 \%$ in cybrids generated using patient mitochondria ${ }^{35}$. The apparent

529 clinical dominance of such low copy number mitochondrial mutations has also been reported in rodent models $9,36,37,38$, and may reflect tissue-specific and/or environmental effects including local bioenergetic

531 demands and levels of antioxidants ${ }^{39,40,41,15}$. Additionally, the basal rate of metabolism, or mass-specific-

532 metabolic rate of mice is seven-fold greater than that of humans, placing correspondingly greater demands

533 on mitochondrial function and energy production especially in high energy demand tissues ${ }^{42}$. Consequently,

534 higher rates of ROS production, major drivers of mitochondrial disease including LS, and predicted to

535 increase with higher mass-specific-metabolic rates, are more likely to destabilize redox homeostasis in

536 mouse models, increasing susceptibility to defective mitochondrial function. Such activities are thought to

537 contribute to the reduced lifespan and increased vulnerability of mice to age-related tumorigenesis relative

538 to humans, both key characteristics of clinical LS. The apparent dominant negative actions of such

539 mitochondrial mutations can account for the persistence of severe phenotypes in our F3 to F6 progeny

540 despite marked dilution of the m.8993T $>\mathrm{G}$ variant in favor of wild type ATP6. Also, in agreement with

541 our previous study, we found no evidence for integration of AAV-encoded ATP6 DNA into the

542 mitochondrial genome (data not shown) and we attribute transgene dilution to low or negligible replication

543 of episomal AAV transgene DNA, as previously reported ${ }^{9}$.

544 Whereas the correlations between disease phenotype, variant m.8993T $>\mathrm{G}$ load and defective ATPase 545 activity, including the markedly higher retinal cell loss associated with variant load in the outer retinal 546 layers of F0-F2 mice are consistent with molecular pathologies reminiscent of clinical MILS/NARP, our 547 demonstration that the disease phenotype was rescued by AAV9 delivery of a wild type ATP6 subunit gene 548 not only supports the link between the disease phenotypes and mutant ATP6 load, but also provides a basis 549 for treatment of LS by gene therapy. By using the same strategy described previously by our group for 550 rescue of $\mathrm{LHON}$ mice $^{9}$, we delivered a mito-targeted wild type ATP6 subunit gene to m.8993T $>\mathrm{G}$ 551 transgenic mice by orbital venous sinus injections of AAV9 to achieve efficient penetration of the blood 552 brain barrier and gene targeting to mitochondria. As also described in the previous study, rescue was robust 
especially when gene delivery was implemented prior to symptom onset. Our molecular analyses of

554 heteroplasmy in rescued mice confirmed the co-presence of variant and rescue ATP6 DNA and protein in

555 rescued mitochondria as well as significantly improved ATPase activity as assessed by in-gel activity of

556 ATP hydrolysis in early rescue tissue samples. Rescued phenotypic parameters of mice containing the

557 m.8993T $>$ G mutation treated at age 3-months included $100 \%$ survival through 15 months without severe

558 symptoms, compared with a 12-month $33 \%$ mortality of non-rescued transgenic mice, significantly

559 improved visual function as well as normalized body weights and resolved cardiomegaly. In addition,

560 histopathology confirmed markedly less vacuolization of brain and spinal cords in rescued animals and 561 reduced cell loss.

562 The absence of animal models that accurately reflect genotypes and corresponding disease phenotypes of 563 mitochondrial diseases, including MILS/NARP have hindered development of effective therapies ${ }^{43}$. 564 Nuclear localization and transcription (allotropic expression) of the ATP6 m.8993T $>$ G mutation followed 565 by cytoplasmic translation and transport into mitochondria was reported to mimic mitochondrial disease in 566 mice ${ }^{44}$. Homoplastic models can be approximated by gene knockout and such models have been used to 567 demonstrate amelioration of disease phenotypes by AAV-based complementation of an Ndufs4 gene 568 knockout in mouse models of $\mathrm{LS}^{4,8}$. Similarly, mutant mtDNA loading of mice with a genetically 569 manipulated heteroplasmic m.5024C $>$ T gene mutation was markedly reduced by AAV9-mediated delivery 570 of mito-targeted TALENs that selectively degraded mutant mtDNA and restored normal levels of the 571 affected mitochondrial alanine tRNA gene product ${ }^{45}$. To our knowledge, studies reported here represent 572 the first to directly target mitochondria with a human m.8993T $>$ G mutation, establish and characterize a 573 disease phenotype that is consistent with clinical presentation of patients with the same mutation, and 574 confirm gene therapy rescue of affected animals by complementation with a similarly targeted AAV9 vector 575 expressing wild type ATP6. The results support further development of gene therapy approaches for 576 MILS/NARP and other mitochondrial diseases perhaps in combination with newer pharmacology based on 577 amelioration of oxidative stress caused by excessive ROS leakage from defective mitochondria and known 
578 to play a central role in cytotoxicity and cell death during mitochondrial disease ${ }^{15,46}$. The studies support

579 and extend our previous work on gene therapy for LHON that are now in clinical development ${ }^{47}$.

Materials and Methods

Ethics statement. This study was carried out in accordance with the recommendations in the Guide for the Care and Use of Laboratory Animals of the National Institutes of Health. All mouse studies were approved by the University of Miami Institutional Animal Care and Use Committee (IACUC).

Plasmids and constructs. A human mitochondrial ATP6 gene fused with a FLAG epitope was synthesized by Genscript, Inc. and cloned in a self-complementary adeno-associated virus (AAV) vector, in which expression was driven by the human mitochondrial heavy strand promoter (HSP). To clone the plasmid scHSP-mtATP6FLAG-mCherry, plasmids sc-HSP-mtATP6 and sc-HSP-ND4-mCherry ${ }^{16}$ were respectively digested by Xba I and BamH I and religated to create sc-HSP-mtATP6FLAG-mCherry, in which mCherry follows the mtATP6FLAG stop codon (see Fig. 1A). The human mutant m.8993 T>G ATP6 was generated by site directed mutagenesis (Quikchange II XL site-directed mutagenesis kit, Stratagene) with substitution of $\mathrm{T}$ to $\mathrm{G}$ at nucleotide position 8993 . The primers used for site directed mutagenesis are listed in Supplementary Table 4. Plasmid AAV9COX8 was constructed by a large fragment polymerase chain reaction (PCR) and seamless cloning of the cytochrome oxidase subunit 8 mitochondrial targeting sequence (COX8) to the $\mathrm{N}$ terminus of VP2 of the AAV9 capsid. The forward primer was designed using the COX8 fragment following VP2 and the reverse primer was the fragment of complementary COX8 following the C terminus of VP1. The primers designed for cloning COX8 to AAV9VP2 are listed in Supplementary Table 5.

Mito-targeted AAV vector production and purification. All mito-targeted AAV vector production and purifications were performed in-house. Mito-targeted AAV2-ATP6FLAG, AAV2-ATP6FLAG-mCherry and AAV2-mutATPFLAG-mCherry were respectively packaged using plasmids sc-HSP-mtATP6FLAG, sc-HSP-mtATP6FLAG-mCherry or sc-HSP-mutATP6FLAG-mCherry, with pVP2COX8 AAV at ratios 
1:1:4. Mito-targeted AAV2/9-ATP6FLAG-mCherry was produced by combining four plasmids: AAV2/9, sc-HSP-ATP6FLAG-mCherry, VP2COX8 and pHelper $(1: 1: 1: 2)$ or three plasmids: AAV2/9COX8, scHSP-ATP6FLAG-mCherry and pHelper (1:1:2). Briefly, 293AAV cells were cultured at $37^{\circ} \mathrm{C}$ to $50 \%-70 \%$ confluency with $10 \%$ heat inactivated (HI) FBS DMEM containing $100 \mathrm{U} / \mathrm{mL}$ of penicillin and $100 \mu \mathrm{g} / \mathrm{mL}$ streptomycin before transfection. Calcium phosphate transfection was performed according to a standard 607 procedure ${ }^{36}$. Vectors were purified by gradient ultracentrifuge and FPLC using a GE AKTA Purifier. The 608 viruses were concentrated, and buffer exchanged in PBS, with a Biomax 100kDa concentrator (Millipore, 609 Billerica, MA). Viral titers were determined by SYBR-Green real-time PCR relative to a standard curve. Viral purity was validated by Coomassie blue stained SDS-PAGE, assayed for sterility, divided into aliquots, and stored at $-80{ }^{\circ} \mathrm{C}$.

Animal Development. Microinjections of mitochondrial-targeted AAV vectors into fertilized oocytes were performed by the UNC transgenic core facility using a standard microinjection protocol ${ }^{37}$. Briefly, hybrid $(\mathrm{C} 57 \mathrm{BL} / 6 \mathrm{~J} \times \mathrm{DBA} / 2 \mathrm{~J}) \mathrm{F} 1$ mice, referred to herein as B6D2F1 mice, were obtained from the Jackson Laboratory for production of fertilized oocytes. Superovulation was stimulated by i.p. injection of B6D2F1 females with 5 IU pregnant mare serum gonadotropin (National Hormone and Peptide Program, National

617 Institute of Diabetes and Digestive and Kidney Diseases) after 46-48 hr. by injection of 5 IU human 618 chorionic gonadotropin (HCG) (Sigma-Aldrich. St Louis, MO). Following HCG injection, females were 619 mated with B6D2F1 stud males, and oocytes were harvested the following day. Approximately 1-2 pL of 620 the MTS-AAV-HSPmutantATP6FLAG + mCherry virus $\left[1.8 \times 10^{12}\right.$ vector genomes $\left.(\mathrm{vg}) / \mathrm{mL}\right]$ was 621 microinjected into fertilized oocytes using a continuous flow injection mode. Surviving eggs were 622 implanted into the ampulla of pseud-pregnant Swiss Webster (Taconic) recipient females. After weaning 623 the resulting offspring were transferred to the University of Miami and analyzed for the presence of the 624 transgene. Viral injections resulted in the offspring listed in Supplementary Table 1.

625 Cell culture and AAV transduction. Homoplasmic m.8993T $>$ G cybrids (NARP cells from Dr. M. Tariq 626 Bhatti) were cultured with high glucose Dulbecco's Modified Eagle Medium (DMEM) (Invitrogen, 
627 Cat.11995) with 10\% HI-FBS and 1\% penicillin and streptomycin containing $4.5 \mathrm{~g} / 1$ glucose, $2 \mathrm{mM}$

628 glutamine and $110 \mu \mathrm{g} / \mathrm{ml}$ sodium pyruvate, and supplemented with $50 \mu \mathrm{g} / \mathrm{ml}$ uridine. $80 \%$ confluent cybrids 629 cells were transduced by mito-targeted AAV9/ATP6FLAG-mCherry $\left(1.8 \times 10^{12} \mathrm{vg} / \mathrm{ml}\right)$ in DMEM without 630 FBS for 2-3hrs, followed by DMEM with 10\%FBS for $\sim 36$ hours and selection in glucose-free media with $63150 \mu \mathrm{g} / \mathrm{ml}$ galactose for 5 days. For electron microscopy (EM), cybrids on gold mesh EM grids were infected 632 with mito-targeted AAV9 containing wild-type ATP6 at MOI of 5000 and examined for AAV particles 26334 hours later without counterstaining.

634 Cell survival assay. Homoplasmic m.8993 $\mathrm{T}>\mathrm{G}$ cybrids in 96-well plates at density 2000 cells per well 635 were infected with mito-targeted AAV containing wild-type ATP6 at multiplicities of infection (MOI) 0, $636500,1000,5000$ and 10,000 for 5hrs in triplicate. After five days of selection in glucose free galactose 637 media, viable cell numbers were counted using a BioRad, TC20.

638 ATP synthesis assay. Luciferin-luciferase assays were used to quantify ATP synthesis by cybrids. 639 Homoplasmic cybrids were seeded in 6-well plates and infected with mito-targeted AAV9-mtATP6FLAG 640 at MOIs $0,500,1000,5000$ and 10,000 followed by selection for 5 days and culture to confluence in normal 641 growth media with $50 \mu \mathrm{g} / \mathrm{ml}$ uridine. Rinsed pellets of $5 \times 10^{5}$ cells were resuspended in ATP synthesis buffer 642 The rate of ATP synthesis of excised tissues was measured by chemiluminescence using a modified 643 luciferin-luciferase assay in digitonin-permeabilized tissues with the complex I substrates malate and 644 pyruvate in real-time using a luminometer (Optocom I; MGM Instruments, Hamden, CT) in a total volume 645 of $200 \mu \mathrm{L}$ that included the following: $160 \mu \mathrm{L}$ of Buffer A (150 mM KCL, $25 \mathrm{mM}$ Tris-HCL, $2 \mathrm{mM}$ EDTA, $646 \quad 0.1 \% \mathrm{BSA}, 10 \mathrm{mM}$ potassium phosphate, $0.1 \mathrm{mM} \mathrm{MgCl}$, $\mathrm{pH} 7.4), 2.5 \mu \mathrm{L}$ of $1 \mathrm{mM}$ Malate in buffer A, 2.5 $647 \mu \mathrm{L}$ of $1 \mathrm{mM}$ Pyruvate in buffer $\mathrm{A}, 5 \mu \mathrm{L}$ of $0.1 \mathrm{mM}$ ADP in Buffer $\mathrm{A}, 5 \mu \mathrm{L}$ of $0.15 \mathrm{mM}$ adenosine 648 pentaphosphate in water, $10 \mu \mathrm{L}$ cells, $10 \mu \mathrm{L}$ of buffer B $(194.4 \mu \mathrm{L}$ of solution $\mathrm{A}, 1.6 \mu \mathrm{L}$ of $100 \mathrm{mM}$ Luciferin 649 in water and $4 \mu \mathrm{L}$ of $1 \mathrm{mg} / \mathrm{ml}$ Luciferase in $0.5 \mathrm{M}$ Tris-acetate $\mathrm{pH} 7.75), 5 \mu \mathrm{L}$ of digitonin $(50 \mu \mathrm{g} / \mathrm{ml})$ with or 650 without $5 \mu \mathrm{L} 0.05 \mathrm{mg} / \mathrm{mL}$ oligomycin in ethanol. Oxidative phosphorylation (OXPHS) was calculated by 
total ATP (without oligomycin) minus the value with oligomycin. ATP and oxidative phosphorylation

652 indices are expressed as units per mg protein.

653 PCR, Sanger DNA sequencing and RT-PCR. Mitochondria were isolated from cybrids or mouse tissues 654 using a dedicated isolation kit according to the manufacturer's instructions (Mitosciences, Cat. MS850 and 655 MS852). Mitochondrial DNA was extracted with a DNeasy Blood \& tissues kit (QIAGEN Cat.69504). Sequences of forward and reverse primers to amplify the 604bp ATP6 fragment were respectively GCT TCA TTC ATT GCC CCC AC, and AGG CGA CAG CAG TTT CTA GG. The 695bp fragment was amplified with the human ATP6 GCT TCA TTC ATT GCC CCC AC as forward primer and Flag CTT GTC GTC ATC GTC TTT GTA GTC as reverse primer. The two sets of primers were used to amplify both human wild-type and mutant ATP6 fragments from cybrids followed by sequencing to confirm the identity. To determine relative contributions of wild type and m.8993T $>$ G mutation ATP6, the signal intensities of the relevant DNA bands separated by agarose gel were measured using Image J. Mitochondrial pellets were solubilized in Native Page Sample buffer with $2.5 \%$ digitonin, incubated on ice for 15 mins, centrifuged at 20,000g for 30 minutes at $4{ }^{\circ} \mathrm{C}$ and the supernatants were aliquoted and stored in $-80^{\circ} \mathrm{C}$. Protein concentration were determined by BCA protein assay, BSA was used as a standard.

667 Proteins separated on precast native PAGE TM 4-16\% Bis-Tris gels from life technologies and processed as 668 per manufacturer's instructions (Cat. BN1002BOX). Fifty $\mu \mathrm{g}$ of mitochondrial protein were used for one 669 dimensional native blue gel Coomassie staining and complex V in gel assays. Transfer membranes were 670 fixed in $8 \%$ acetic acid for $15 \mathrm{~min}$, blocked for $1 \mathrm{hr}$. and detected respectively with FLAG (1:1000, Origen, 671 Cat. No. TA100011), mCherry antibody (1:500, Abcam, Cat. No. ab125096), mt-ATP6 antibody (1:1000, 672 Cat. PA5-103625) and ATP5A antibodies (1:1000, Abcam, ab28777). For 2-D immunoblotting, 200 $\mu \mathrm{g}$ of 673 mitochondrial protein were used and strips from the first dimension were cut and denatured in 50mM DDT 674 one hour before running the second dimensional gel. The gels were transferred to PVDF membrane and 675 detected respectively with FLAG, mCherry, ATP5A and mt-ATP6 antibodies. For complex V in-gel 
activity, washed gels were incubated in $50 \mathrm{mM}$ Tris $\mathrm{pH} 8.6$ for one hour followed by incubation with 35

$677 \mathrm{mM}$ Tris, $270 \mathrm{mM}$ glycine, $14 \mathrm{Mm} \mathrm{MgSO}_{4}, 5 \mathrm{mM}$ ATP and $0.2 \% \mathrm{~Pb}\left(\mathrm{NO}_{3}\right)_{2}$ at $37^{\circ} \mathrm{C}$ for 3 hrs. or overnight ${ }^{48}$.

678 After washing in water, gels were scanned and photographed. Western blot membranes were respectively 679 reacted with anti-FLAG, anti-mCherry, and anti-VDAC/Porin antibodies (Abcam, Cat.ab17434) with AAV 680 transduced samples respectively stained by cox8 and AAV antibodies (anti-cox 8 antibody, Cat. LS681 C664745; anti-AAV B1, Progen, Cat.65158, mouse monoclonal B1). ECL chemiluminescence was used to 682 visualize the bands using a FUJI400 imager.

683 Mass spectrometry. Mass spectrometry (MS) was performed by the Harvard University Mass 684 Spectrometry core facility. Protein bands were excised from 1 and 2-D blue native gels, digested with trypsin 685 and subject to MS. Briefly, capillary reverse phase HPLC separation of protein digests were performed on 686 a 10-cm 75-m inner diameter PepMap C18 column (LC Packings, San Francisco, CA) in combination with 687 an in-house capillary HPLC system operated at a flow rate of $200 \mathrm{nl} / \mathrm{min}$. In-line mass spectrometric analysis 688 of the column eluate was accomplished by a quadrupole ion trap instrument (LCQ; ThermoFinnigan, San 689 Jose, CA) equipped with a nanoelectrospray source. Fragment ion data generated by data-dependent 690 acquisition via the LCQ were searched against the NCBI nr sequence database using the SEQUEST (Thermo 691 Finnigan) and Mascot (Matrix Science, Boston) database search engines. In general, the score for SEQUEST 692 protein identification was considered significant when $\mathrm{dCn}$ was equal to 0.08 or greater and the cross693 correlation score was greater than 2.2. MASCOT probability-based MOWSE scores above the default 694 significant value were considered for protein identification in addition to validation by manual interpretation 695 of the tandem mass spectrometry data.

696 Magnetic Resonance Imaging (MRI). High-resolution (4.7T magnet) MRI (Oxford Instruments Oxford, 697 UK) was performed on mildly symptomatic F0 A6 mice maintained under 1.5 to 2\% isoflurane anesthesia. 698 All animals were placed in a prone position with heads held firmly by a purpose-built head coil. T2-weighted 699 images of the brain were acquired. 
701 or unstained retina, spinal cord, brain of transgenic A6 mice were cut into 8-18 $\mu \mathrm{M}$ and placed on director 702 slides. Laser capture microdissection was performed using a Leica LMD6500. Mitochondrial DNA was 703 extracted from mCherry positive cells. Taqman RT-PCR assays were performed to quantify human and 704 mouse mitochondrial ATP6 copy numbers with mtATP6 plasmid DNA as a standard curve (Thermo 705 scientific mouse mtATP6: Cat. Mm03649417_g1; human mtATP6: Hs02596862_g1).

A6 mouse phenotype and histopathology. Phenotypes of A6 mice including weight loss, off balance, 707 ocular defects, hunching, seizures, paralysis and mortality were observed and recorded over six generations. A6 mice were weighed and anesthetized using ketamine/xylazine (i.p. $80 \mathrm{mg} / \mathrm{kg}$ and $20 \mathrm{mg} / \mathrm{kg}$ body weight). Tissues of A6 mice including brain, spinal cord, eyes, optic nerve, muscle, spleen, liver and lung were fixed in $10 \%$ formalin. H\&E staining was performed by the histology core lab at the University of Miami. Images

711 were captured using a Leica fluorescence microscope (Buffalo Grove, IL).

712 Brain sections and TEM. Brains were immersion fixed in 2\% paraformaldehyde, $2 \%$ glutaraldehyde in 0.1 713 M PBS buffer, and post fixed in 1\% osmium tetroxide, $0.1 \mathrm{M}$ sodium cacodylate-HCl buffer, $\mathrm{pH}$ 7.4. Tissues 714 were dehydrated through ethanol to propylene oxide, infiltrated, and embedded in epoxy resin. Semithin 715 longitudinal sections $(0.5 \mu \mathrm{m}$ to $1 \mu \mathrm{m})$ of the brain were stained with toluidine blue and placed on nickel 716 grids for ultrastructural examination using a JEOL JEM 1400 TEM and GATAN camera (Orius SC200 717 CCD). Image quantifications were performed by image J.

718 Rotarod assay. A6 and wild-type C57BL/6 mice motor and balance were evaluated using an LE8500 719 rotarod (Panlab, Spain) as per manufacturer's instructions. Time to fall was recorded on the instrument 720 screen with a starting speed of $4 \mathrm{rpm}$ that was gradually increased. Each mouse was given one trial 721 (maximum time 10 minutes). The time of latency-to-fall and speed were recorded for each trial.

722 Pattern and flash electrophysiology. Mice were weighed, anesthetized with ketamine/xylazine (i.p. 80 $723 \mathrm{mg} / \mathrm{kg}$ and $20 \mathrm{mg} / \mathrm{kg}$ body weight $)$ placed on a heating pad $\left(37^{\circ} \mathrm{C}\right)$ and restrained using a bit bar and a nose 
724 holder with unobstructed vision. A pattern electroretinogram (PERG) electrode, diameter of 0.25mm, was 725 placed on the corneal surface by means of a micromanipulator and positioned to encircle the pupil without 726 limiting the field of view. Reference and ground electrodes were stainless steel needles inserted under the 727 skin of the scalp and tail, respectively. Before recording, a small drop of balanced salt solution was topically 728 applied to the cornea to prevent drying. A visual stimulus of contrast-reversing $(1 \mathrm{~Hz}, 2$ reversal/s) horizontal 729 bars generated by a programmable graphic card (VSG-Cambridge Research Systems, Rochester, UK) on a 730 cathode-ray tube (CRT) display (Sony Multiscan 500) was aligned with the projection of the pupil. At a 731 viewing distance of $15 \mathrm{~cm}$, the stimulus field covered an area of 69.4 x $63.4 \mathrm{deg}$. Patterns had a fixed mean 732 luminance of $50 \mathrm{~cd} / \mathrm{m} 2$. Retinal signals were amplified (10,000-fold) and band-pass filtered (1-30 Hz). Three 733 consecutive responses to 600 contrast reversals each were recorded. The responses were superimposed to 734 check for consistency and then averaged. To obtain a corresponding index of outer retinal function, flash 735 electroretinogram (FERG) was also recorded in response to strobe flashes of $20 \mathrm{~cd} / \mathrm{m}^{2} / \mathrm{s}$ superimposed on a 736 steady background light of $12 \mathrm{~cd} / \mathrm{m}^{2}$ and presented within a Ganzfeld bowl. Under these conditions, rod 737 activity is largely suppressed while cone activity is minimally suppressed. Averaged PERG and FERG were 738 automatically analyzed and evaluated the major positive and negative waves by Sigma Plot (Systat software 739 Inc., San Jose, CA).

740 Virus administration. All animal procedures were conducted in facilities accredited by the Association for 741 Assessment and Accreditation of Laboratory Animal Care and in accordance with the University of Miami 742 Institutional Animal Care and Use Committee policies. Death was not an intentional endpoint and mice 743 were euthanized when moribund. Mice were sedated by inhalation with $1.5 \%$ to $2 \%$ isoflurane. A local 744 anesthetic (proparacaine $\mathrm{HCl}$ ) was applied topically to the cornea and then a 32gauge needle attached to the 745 Hamilton syringe. Visual and motor functions were assessed and recorded by PERG, Flash ERG and Rotarod 746 assays. Mice samples were collected and analyzed as described above.

747 Statistical analysis. All data plotted with error bars are expressed as the mean \pm SEM. The $P$ values were 748 generated by analyzing data with student's t-tests, one-way ANOVA or two-way ANOVA using GraphPad 
Prism 7 software. Cumulative proportional survival rate statistical analysis was performed by Kaplan-Meier

analyses and statistical analyses were performed with log-rank test.

751

752

753

754

755

756

757

758

759

760

761

762

763

764

765

766

767

768

769

770

771

772

773

774

775

776

777

778

779

780

781

782

783

784

785

786

787

788

789

790

791

792

\section{References}

1. Gerards, M., Sallevelt, S.C. \& Smeets, H.J. Leigh syndrome: Resolving the clinical and genetic heterogeneity paves the way for treatment options. Mol Genet Metab 117, 300-12 (2016).

2. Darin, N., Oldfors, A., Moslemi, A.R., Holme, E. \& Tulinius, M. The incidence of mitochondrial encephalomyopathies in childhood: clinical features and morphological, biochemical, and DNA abnormalities. Ann Neurol 49, 377-83 (2001).

3. Ruhoy, I.S. \& Saneto, R.P. The genetics of Leigh syndrome and its implications for clinical practice and risk management. App/ Clin Genet 7, 221-34 (2014).

4. Reynaud-Dulaurier, R. et al. Gene replacement therapy provides benefit in an adult mouse model of Leigh syndrome. Brain 143, 1686-1696 (2020).

5. Kirby, D.M. et al. Respiratory chain complex I deficiency: an underdiagnosed energy generation disorder. Neurology 52, 1255-64 (1999).

6. Quintana, A. et al. Fatal breathing dysfunction in a mouse model of Leigh syndrome. J Clin Invest 122, 2359-68 (2012).

7. Kruse, S.E. et al. Mice with mitochondrial complex I deficiency develop a fatal encephalomyopathy. Cell Metab 7, 312-20 (2008).

8. Di Meo, I., Marchet, S., Lamperti, C., Zeviani, M. \& Viscomi, C. AAV9-based gene therapy partially ameliorates the clinical phenotype of a mouse model of Leigh syndrome. Gene Ther 24, 661-667 (2017).

9. $\mathrm{Yu}, \mathrm{H}$. et al. Consequences of zygote injection and germline transfer of mutant human mitochondrial DNA in mice. Proc Natl Acad Sci U S A 112, E5689-98 (2015).

10. $\mathrm{Yu}, \mathrm{H}$. et al. Gene delivery to mitochondria by targeting modified adenoassociated virus suppresses Leber's hereditary optic neuropathy in a mouse model. Proc Natl Acad Sci U S A 109, E1238-47 (2012).

11. Yu, H. et al. Mutant NADH dehydrogenase subunit 4 gene delivery to mitochondria by targeting sequence-modified adeno-associated virus induces visual loss and optic atrophy in mice. Mol Vis 18, 1668-83 (2012).

12. Rahman, S. Emerging aspects of treatment in mitochondrial disorders. J Inherit Metab Dis 38, 641-53 (2015).

13. Silva-Pinheiro, P., Cerutti, R., Luna-Sanchez, M., Zeviani, M. \& Viscomi, C. A Single Intravenous Injection of AAV-PHP.B-hNDUFS4 Ameliorates the Phenotype of Ndufs4 (-/-) Mice. Mol Ther Methods Clin Dev 17, 1071-1078 (2020).

14. Al Khazal, F. et al. A conditional mouse model of complex II deficiency manifesting as Leigh-like syndrome. FASEB J 33, 13189-13201 (2019).

15. Jain, I.H. et al. Leigh Syndrome Mouse Model Can Be Rescued by Interventions that Normalize Brain Hyperoxia, but Not HIF Activation. Cell Metab 30, 824-832 e3 (2019).

16. El-Desouky, S., Taalab, Y.M., El-Gamal, M., Mohamed, W. \& Salama, M. Animal Model for Leigh Syndrome. Methods Mol Biol 2011, 451-464 (2019).

17. Maul, G.G., Rovera, G., Vorbrodt, A. \& Abramczuk, J. Membrane fusion as a mechanism of simian virus 40 entry into different cellular compartments. J Virol 28, 936-44 (1978).

18. Szabo, l. et al. Double-stranded DNA can be translocated across a planar membrane containing purified mitochondrial porin. FASEB J 12, 495-502 (1998). 
19. Weber-Lotfi, F. et al. Developing a genetic approach to investigate the mechanism of mitochondrial competence for DNA import. Biochim Biophys Acta 1787, 320-7 (2009).

20. Lightowlers, R.N. \& Chrzanowska-Lightowlers, Z.M. Terminating human mitochondrial protein synthesis: a shift in our thinking. RNA Biol 7, 282-6 (2010).

21. Grimm, D., Kern, A., Rittner, K. \& Kleinschmidt, J.A. Novel tools for production and purification of recombinant adenoassociated virus vectors. Hum Gene Ther 9, 2745-60 (1998).

22. Bonfante, E., Koenig, M.K., Adejumo, R.B., Perinjelil, V. \& Riascos, R.F. The neuroimaging of Leigh syndrome: case series and review of the literature. Pediatr Radiol 46, 443-51 (2016).

23. Sinko, G., Garzuly, F. \& Kalman, B. Striking pathology in Leigh syndrome associated with the MTATP6 T8993G mutation. Pediatr Neurol 51, 585-6 (2014).

24. Hayashi, N., Geraghty, M.T. \& Green, W.R. Ocular histopathologic study of a patient with the T 8993-G point mutation in Leigh's syndrome. Ophthalmology 107, 1397-402 (2000).

25. Siegmund, S.E. et al. Three-Dimensional Analysis of Mitochondrial Crista Ultrastructure in a Patient with Leigh Syndrome by In Situ Cryoelectron Tomography. iScience 6, 83-91 (2018).

26. Leung, D.G. et al. Mitochondrial DNA Deletions With Low-Level Heteroplasmy in Adult-Onset Myopathy. J Clin Neuromuscul Dis 19, 117-123 (2018).

27. Ng, Y.S. et al. MT-ND5 Mutation Exhibits Highly Variable Neurological Manifestations at Low Mutant Load. EBioMedicine 30, 86-93 (2018).

28. Hirose, M. et al. Low-level mitochondrial heteroplasmy modulates DNA replication, glucose metabolism and lifespan in mice. Sci Rep 8, 5872 (2018).

29. D'Aurelio, M., Vives-Bauza, C., Davidson, M.M. \& Manfredi, G. Mitochondrial DNA background modifies the bioenergetics of NARP/MILS ATP6 mutant cells. Hum Mol Genet 19, 374-86 (2010).

30. Dayton, R.D., Wang, D.B. \& Klein, R.L. The advent of AAV9 expands applications for brain and spinal cord gene delivery. Expert Opin Biol Ther 12, 757-66 (2012).

31. Ganetzky, R.D. et al. MT-ATP6 mitochondrial disease variants: Phenotypic and biochemical features analysis in 218 published cases and cohort of 14 new cases. Hum Mutat 40, 499-515 (2019).

32. van den Ameele, J., Li, A.Y.Z., Ma, H. \& Chinnery, P.F. Mitochondrial heteroplasmy beyond the oocyte bottleneck. Semin Cell Dev Biol 97, 156-166 (2020).

33. Stendel, C. et al. Delineating MT-ATP6-associated disease: From isolated neuropathy to early onset neurodegeneration. Neurol Genet 6, e393 (2020).

34. Chinnery, P.F., Andrews, R.M., Turnbull, D.M. \& Howell, N.N. Leber hereditary optic neuropathy: Does heteroplasmy influence the inheritance and expression of the G11778A mitochondrial DNA mutation? Am J Med Genet 98, 235-43 (2001).

35. Sacconi, S. et al. A functionally dominant mitochondrial DNA mutation. Hum Mol Genet 17, 1814-20 (2008).

36. Qi, X., Sun, L., Lewin, A.S., Hauswirth, W.W. \& Guy, J. The mutant human ND4 subunit of complex I induces optic neuropathy in the mouse. Invest Ophthalmol Vis Sci 48, 1-10 (2007).

37. Guy, J. et al. Rescue of a mitochondrial deficiency causing Leber Hereditary Optic Neuropathy. Ann Neurol 52, 534-42 (2002).

38. Ellouze, S. et al. Optimized allotopic expression of the human mitochondrial ND4 prevents blindness in a rat model of mitochondrial dysfunction. Am J Hum Genet 83, 373-87 (2008).

39. Lin, C.S. et al. Mouse mtDNA mutant model of Leber hereditary optic neuropathy. Proc Natl Acad Sci U S A 109, 20065-70 (2012).

40. Pilz, Y.L., Bass, S.J. \& Sherman, J. A Review of Mitochondrial Optic Neuropathies: From Inherited to Acquired Forms. J Optom 10, 205-214 (2017).

41. Ferrari, M. et al. Hypoxia treatment reverses neurodegenerative disease in a mouse model of Leigh syndrome. Proc Natl Acad Sci U S A 114, E4241-E4250 (2017). 
42. Demetrius, L. Of mice and men. When it comes to studying ageing and the means to slow it down, mice are not just small humans. EMBO Rep 6 Spec No, S39-44 (2005).

43. Kauppila, J.H.K. et al. A Phenotype-Driven Approach to Generate Mouse Models with Pathogenic mtDNA Mutations Causing Mitochondrial Disease. Cell Rep 16, 2980-2990 (2016).

44. Dunn, D.A. \& Pinkert, C.A. Allotopic expression of ATP6 in the mouse as a transgenic model of mitochondrial disease. Methods Mol Biol 1265, 255-69 (2015).

45. Bacman, S.R. et al. MitoTALEN reduces mutant mtDNA load and restores tRNA(Ala) levels in a mouse model of heteroplasmic mtDNA mutation. Nat Med 24, 1696-1700 (2018).

46. Jain, I.H. et al. Hypoxia as a therapy for mitochondrial disease. Science 352, 54-61 (2016).

47. Koilkonda, R.D. et al. Safety and effects of the vector for the Leber hereditary optic neuropathy gene therapy clinical trial. JAMA Ophthalmol 132, 409-20 (2014).

48. Fontanesi, F., Diaz, F. \& Barrientos, A. Evaluation of the mitochondrial respiratory chain and oxidative phosphorylation system using yeast models of OXPHOS deficiencies. Curr Protoc Hum Genet Chapter 19, Unit19 5 (2009).

Acknowledgements The work was conceived, planed and directed by Dr. John Guy who died as this manuscript was being revised. We remember John as a brilliant biomedical scientist with a lifelong dedication to mitochondrial disease therapy. We thank Dr. Hong Yu for generously providing the sc-HSPATP6 plasmid, William J. Feuer and Dr. Chuanhui Dong for expert advice on statistical analyses.

Funding Support This study was supported by the National Eye Institute R01EY027414 (Guy), R01EY 017141 (Guy), R24EY028764 (Webster), R43EY031238 (Webster), P30 EY014801 Bascom Palmer Eye Institute Core Grant (Porciatti) and the University of Miami Electron Microscopy Core IS100D012061 (Salas). Institutional support to BPEI was from a Research to Prevent Blindness Unrestricted Grant (GR004596) and an NEI Center Core Grant (EY014801).

Author contributions H. J. Y was responsible for creating and characterizing the LS mouse model, rescue vector construction and production, data compilation, data analysis, and manuscript writing. K.A.W contributed data analysis and manuscript writing. M.T.B., W.W.H., and A.S.L., proof reading and provided reagents. J. G. initiated, designed and directed the study, performed hands on application of mouse MRI, EM, and AAV injections, and contributed importantly to writing and communicating the first version of the manuscript. 


\section{Supplementary Files}

This is a list of supplementary files associated with this preprint. Click to download.

- Video6.avi.zip

- Video7.avi.zip

- Video3.mp4.mp4

- Video2Seizure.avi.zip

- Video5AfterTREpara.wmv.zip

- Video1Para.wmv.zip

- Video4BeforeTxPara.avi.zip

- Yuanetal.Supplementaryinfo.pdf 